\title{
Effect of Wiggler Insertions on the Single-Particle Dynamics of the NLC Main Damping Rings*
}

\author{
M. Venturini \\ Lawrence Berkeley National Laboratory, \\ Berkeley, CA, 94720.
}

\begin{abstract}
As they are expected to occupy a large portion of the lattice, wiggler insertions will introduce significant linear and nonlinear perturbations to the single-particle dynamics in the NLC Main Dampig Rings (MDR). The nonlinearities are of particular concern as a sufficiently large Dynamic Aperture (DA) is required for high injection efficiency. The main content of this report is a study of the wigglers impact on the DA of the NLC-MDR latest lattice design. The particle dynamics is modelled by transfer maps calculated by integration through the wiggler fields. For field representation we employed a 3D multipole expansion derived from the field data that were obtained with the aid of a magnet design code. Additional contents of this paper include an investigation of a simplified model of wiggler consisting of a sequence of standard magnet elements (where thin octupoles are used to represent the dominant nonlinearities) and the suggestion of a possible correction scheme to compensate the wiggler nonlinearities.
\end{abstract}

*Work supported by Department of Energy contract DE-AC03-76SF00098. 


\section{Introduction}

In the damping rings of the next generation of linear colliders most radiation loss will take place in the wiggler insertions rather than bending magnets. As they occupy a large portion of the lattice, wigglers introduce significant linear and nonlinear perturbation to beam dynamics ${ }^{\dagger}$. An accurate assessment of these effects is desirable in order to guarantee a proper tuning of the linear lattice and determine the impact on the Dynamic Aperture (DA) - a critical quantity because the large power carried by the circulating beams demands a very high injection efficiency and places a severe constraint on allowable losses.

In the current design $[1,2]$ of the Next Linear Collider (NLC) Main Damping Ring (MDR) lattice the total length of the wiggler insertions is $61.6 \mathrm{~m}$ out of a circumference of about $300 \mathrm{~m}$ - an increase of $15 \mathrm{~m}$ from the previous design. The change was needed to enlarge the momentum compaction as a way to contain the effects of collective instabilities. As a larger momentum compaction was achieved by longer bends with smaller magnetic field, additional wigglers were required in order to compensate for the smaller radiation loss in the bendings and preserve the desired damping.

A previous study $[3,4]$ showed that the effects of the wiggler nonlinearities in the earlier design of the NLC MDR lattice while noticeable did not introduce unacceptable degradation of the DA. The study made use of a suitable fitting of the magnetic field in the wiggler midplane and a simplified symplectic integrator for tracking the orbits of individual particles. A similar conclusion was reached after a first investigation of the new lattice [1] that was carried out with the wigglers modelled as sequences of standard elements (combined function dipoles, thin octupoles, and drifts). The parameters for these standard elements were tuned in such a way as to reproduce the same horizontal and vertical kicks through a wiggler period as obtained from the more accurate symplectic integrator technique employed earlier.

In this paper we report on a further study on the new lattice using a method that makes some improvements on the field representation and avoids some of the simplifications in the integrator mentioned above. The method was first introduced in [5] and originally applied to study fringe-effects in superconducting quadrupoles but is sufficiently general to be extended with few modifications to arbitrary magnetic devices. It makes use of one component of the magnetic field defined on a surface surrounding the beam reference orbit and contained within the aperture of the wiggler. Because of the Maxwell equations this information is sufficient for determining the complete magnetic field within the entire aperture of the device. Specification of the magnetic field could come from direct measurement or numerical calculation done with the aid of one of the 3D codes that are customarily used in wiggler design. As we shall see, this method has certain smoothing properties that make the field reconstruction within the outer surface relatively insensitive to possible noise present in the magnetic field data. For this reason, it is preferable to techniques that use magnetic field data defined in the wiggler midplane.

If the reference orbit does not deviate much from a straight line a convenient choice for the outer surface is that of a cylinder. Then, in cylindrical coordinates the magnetic

${ }^{\dagger}$ While wigglers can also significantly affect the beam dynamics by exciting collective instabilities (through emission of coherent synchrotron radiation), in this study we are only concerned with single-particle effects. 
field is best described in terms of a "multipole" expansion, yielding a representation that is particularly suitable for perturbative calculations of the beam dynamics. The relationship between the coefficients of this expansion and the magnetic field data can be easily worked out. Equipped with knowledge of the fields one can integrate the equations of motion for the orbits of individual particles or, more efficiently, calculate the transfer map throughout the entire device or portions of it, through the desired order. The transfer maps so calculated can then be concatenated with those relative to the other machine elements to study the lattice or preform tracking.

In the present study we used the code MARYLiE [6] which has the built-in capability of calculating transfer maps through arbitrary lattice devices upon specification of the corresponding Hamiltonian. We implemented our method in an added-on user-defined routine that reads in the magnetic field data from an external file and extracts the information required to evaluate the Hamiltonian. Because the calculation is carried out in a canonical framework an intermediate step is devoted to determining the vector potential. We mostly used the released 3.0 version of MARYLIE, which allows for map calculations through $3^{\text {rd }}$ order (in the dynamical variable expressing deviation from the reference orbit).

The main result of this Paper - a calculation of the DA for the NLC MDR lattice in the presence of wiggler - is reported in Sec. 10. The estimates of the DA we found are about consistent with those from previous studies, which were obtained with different methods. A degradation of the DA is observed but - at least for on-momentum particles - appears to remain close to the targeted values of the machine acceptance (at injection the NLC MDR's should be capable of accommodating a beam with $150 \mathrm{~mm}$-mrad normalized emittance in both transverse planes and a full width $\pm 1 \%$ energy spread). The DA degradation off-momentum is of concern as well as the question of whether the calculated DA will be preserved in longer term tracking. These are issues that will be the focus of our next investigations.

The rest of the paper is organized as follows. After reporting in Sec. 2 the formalism of a 3D field multipole expansion for the scalar potential, magnetic fields and vector potential, in Sec. 3 we work out the relationships between the magnetic field data and such a field expansion. Sec. 4 contains an analysis of the magnetic field map for one period of the NLC-MDR wigglers, based on an existing preliminary design for the insertions [7]. For comparison we also report an analysis of the field data relative to one period of the wiggler of the TESLA damping ring [8]. An outline of transfer map calculation is presented in Sec. 5, while in Sec. 5.1 and 5.2 we discuss a simplified model of dynamics and give approximate analytical expressions for the transverse kicks experienced by particles crossing a wiggler period. The numerical evaluation of those kicks is then reported in Sec. 7. Finally, Section 8 is devoted to comparing the more accurate calculation of transfer maps discussed so far with one in which the wigglers are modelled using standard elements (combined function dipoles, octupoles), while in Sec. 9 we suggest a possible strategy for correcting the undesired wiggler nonlinearities based on the results of Section 8 . 


\section{Multipole Expansion for the Scalar Potential and Generalized Gradients}

Although we are ultimately interested in the expression for the vector potential, the description of the magnetic field in a current-free region is most conveniently carried out in terms of a scalar potential $\psi$, with $\boldsymbol{B}=\nabla \psi,{ }^{\ddagger}$ obeying the Laplace equation:

$$
\nabla^{2} \psi=0
$$

We choose a cylindrical coordinate system and orient the $z$-axis along the longitudinal axis of the wiggler. In this Paper we will neglect end effects and only consider the fields over one wiggler period $\lambda_{w}$. The most general solution of (1) regular at the origin $\rho=0$ and periodic in $z$, can be expanded in terms of the eigenfunctions of the operator $\partial^{2} / \partial \phi^{2}$ as

$$
\psi=\sum_{m=0}^{\infty} \psi_{m, s}(\rho, z) \sin m \phi+\psi_{m, c}(\rho, z) \cos m \phi .
$$

where $\psi_{m, s}$ and $\psi_{m, c}$ have the following Fourier-series representation involving the modified Bessel functions $I_{m}$

$$
\begin{aligned}
& \psi_{m, s}(\rho, z)=\sum_{p=-\infty}^{\infty} e^{2 \pi i p z / \lambda_{w}} I_{m}\left(\frac{2 \pi p}{\lambda} \rho\right) b_{m, p}, \\
& \psi_{m, c}(\rho, z)=\sum_{p=-\infty}^{\infty} e^{2 \pi i p z / \lambda_{w}} I_{m}\left(\frac{2 \pi p}{\lambda} \rho\right) a_{m, p},
\end{aligned}
$$

and $a_{m, p}$ and $b_{m, p}$ are arbitrary coefficients.

Equation (2) is usually referred to in accelerator literature as a 3D 'multipole expansion'. The integer $m$ is the order of the multipole. For example, $m=0$ corresponds to a pure solenoid, $m=1$ to a dipole, $m=2$ to a quadrupole, and so on. The solenoidal field component is described by the component $\psi_{m=0, c}$, and ordinarily should not appear in the field expansion for a wiggler. The 'sin-like' and 'cos-like' terms (2) correspond to the 'normal' and 'skew' components. Only normal components are contained in the fields of ideal errorfree planar wigglers (the ones of interest in this Paper). In contrast, the field representation of helical wigglers would include skew components as well. In the following the formulae we report will apply when only normal field components are present. The multipole expansion can be easily converted into a power series in the radial variable $\rho$ by using the Taylor expansion for $I_{m}(x)$ :

$$
I_{m}(x)=\sum_{\ell=0} \frac{1}{\ell !(\ell+m) !}\left(\frac{x}{2}\right)^{2 \ell+m} .
$$

Upon insertion of the Taylor series (5) into the expressions (3) and (4), and inversion in the order of the double summation we obtain the 3D multipole expansion in the form that is usually reported in the literature

\footnotetext{
${ }^{\ddagger}$ Notice our convention about the $+\operatorname{sign}$ in the definition of the scalar potential.
} 


$$
\psi_{m}(\rho, z)=\sum_{\ell=0}^{\infty}(-1)^{\ell} \frac{m !}{2^{2 \ell} \ell !(\ell+m) !} C_{m}^{[2 \ell]}(z) \rho^{2 \ell+m}
$$

where (for $m \neq 0$ ) the functions $C_{m}^{[2 \ell]}(z)$ are defined by

$$
C_{m}^{[2 \ell]}(z)=\frac{(-1)^{\ell}}{\sqrt{2 \pi}} \frac{1}{2^{m} m !} \sum_{p=-\infty}^{\infty} e^{2 \pi i p z / \lambda_{w}}\left(\frac{2 \pi p}{\lambda_{w}}\right)^{2 \ell+m} b_{m, p} .
$$

Observe that $C_{m}^{[2 \ell+2]}(z)=\frac{d^{2}}{d z^{2}} C_{m}^{[2 \ell]}(z)$. That is, for each $m$ the coefficients of the series (6) can be obtained by successive differentiation of the functions $C_{m}(z) \equiv C_{m}^{[0]}(z)$. We will refer to the functions $C_{m}(z)$ as 'generalized gradients'. The corresponding expressions for the magnetic field in cylindrical coordinates $(m \neq 0)$ are

$$
\begin{aligned}
& B_{\rho}=\sum_{m=1}^{\infty} \sum_{\ell=0}^{\infty}(-1)^{\ell} \frac{m !(2 \ell+m)}{2^{2 \ell} \ell !(\ell+m) !} C_{m}^{[2 \ell]}(z) \rho^{2 \ell+m-1} \sin m \phi, \\
& B_{\phi}=\sum_{m=1}^{\infty} \sum_{\ell=0}^{\infty}(-1)^{\ell} \frac{m !}{2^{2 \ell} \ell !(\ell+m) !} m C_{m}^{[2 \ell]}(z) \rho^{2 \ell+m-1} \cos m \phi, \\
& B_{z}=\sum_{m=1}^{\infty} \sum_{\ell=0}^{\infty}(-1)^{\ell} \frac{m !}{2^{2 \ell} \ell !(\ell+m) !} C_{m}^{[2 \ell+1]}(z) \rho^{2 \ell+m} \sin m \phi .
\end{aligned}
$$

Having characterized the magnetic field in terms of the multipole expansion of the scalar potential, we want to use the generalized gradients to determine the vector potential. A possible way to proceed is to make an ansatz for the vector potential in the form

$$
\begin{aligned}
& A_{\rho}(\rho, \phi, z)=\sum_{m} A_{\rho}^{(m)}(\rho, z) \cos m \phi \\
& A_{\phi}(\rho, \phi, z)=\sum_{m} A_{\phi}^{(m)}(\rho, z) \cos m \phi \\
& A_{z}(\rho, \phi, z)=\sum_{m} A_{z}^{(m)}(\rho, z) \cos m \phi
\end{aligned}
$$

and use the relationship $\nabla \psi=\nabla \times \boldsymbol{A}$ to determine the coefficients $A_{\rho}^{(m)}, A_{\phi}^{(m)}, A_{z}^{(m)}$ in terms of $\psi_{m}$. For the $A_{\phi}=0$ choice of the gauge we have $A_{\phi}^{(m)}=0$ and

$$
A_{z}^{(m)}=\frac{\rho}{m} \frac{\partial}{\partial \rho} \psi_{m}, \quad A_{\rho}^{(m)}=-\frac{\rho}{m} \frac{\partial}{\partial z} \psi_{m}
$$

For an ideal (error-free) planar wiggler (in which only odd-number multipoles are present) the vector-potential multipole expansion through $6^{\text {th }}$ in $\rho$ reads

$$
\begin{aligned}
& A_{\rho}=\left(\rho^{2} C_{1}-\frac{\rho^{4}}{8} C_{1}^{[3]}+\frac{\rho^{6}}{192} C_{1}^{[5]}\right) \cos \phi+\left(\frac{\rho^{4}}{3} C_{3}^{[1]}-\frac{\rho^{6}}{48} C_{3}^{[3]}\right) \cos 3 \phi+\frac{\rho^{6}}{5} C_{5}^{[1]} \cos 5 \phi, \\
& A_{z}=-\left(\rho C_{1}-\frac{3 \rho^{2}}{8} C_{1}^{[2]}+\frac{5 \rho^{4}}{192} C_{1}^{[4]}\right) \cos \phi-\left(\rho^{3} C_{3}-\frac{5 \rho^{5}}{48} C_{3}^{[2]}\right) \cos 3 \phi-\rho^{5} C_{5} \cos 5 \phi .
\end{aligned}
$$




\section{Calculation of Generalized Gradients from Numeri- cal Field Data}

Knowledge of one component of the magnetic field on the surface of a cylinder is sufficient to determine the entire field in the current-free region both inside and outside that surface. Suppose the the radial component is known and given in terms of Fourier series in the azimuthal angle (again we are assuming that only the normal components of the filed are present)

$$
B_{\rho}(\rho=R, \phi, z)=\sum_{m=0}^{\infty} B_{m}(R, z) \sin (m \phi) .
$$

First, we calculate $B_{\rho}=\frac{\partial}{\partial \rho} \psi$ at $\rho=R$ from (2), (3) and then compare the resulting expression with (13) to determine the coefficients $b_{m, p}$

$$
b_{m, p}=\frac{\lambda_{w}}{2 \pi p} \frac{\tilde{B}_{m, p}}{I_{m}^{\prime}\left(2 \pi p R / \lambda_{w}\right)},
$$

where $\tilde{B}_{m, p}$ are the Fourier integrals of $B_{m}(R, z)$

$$
\tilde{B}_{m, p}=\frac{1}{\lambda_{w}} \int_{0}^{\lambda_{w}} d z e^{-i 2 \pi p z / \lambda_{w}} B_{m}(R, z),
$$

Finally, the expression (14) for $b_{m, p}$ can be inserted into the definition of the generalized gradients (7) to yield

$$
C_{m}^{[k]}(z)=\frac{1}{2^{m} m !} \sum_{p=-\infty}^{\infty} i^{k} \frac{\left(2 \pi p / \lambda_{w}\right)^{m+k-1}}{I_{m}^{\prime}\left(2 \pi p R / \lambda_{w}\right)} e^{i 2 \pi p z / \lambda_{w}} \tilde{B}_{m, p} .
$$

Because the Bessel functions $I_{m}(x)$ increase exponentially for large $x$ they provide an effective high-frequency filter in the evaluation of the generalized gradients (16). Their presence has the favorable effect of softening the numerical noise possibly present in the magnetic field data [5]. This low-pass filtering is enhanced by choosing a value for the radius $R$ (appearing in the argument of the Bessel function) as large as possible. It is this smoothing property that makes the field representation advocated here preferable to the one that relies on using magnetic field data from the wiggler mid-plane. In this other case errors in the magnetic field data are not smoothened but are, in fact, magnified when one tries to evaluate the fields away from the mid-plane.

\section{Field Analysis for the NLC-MDR and Tesla Wiggler}

In this Section we report the field analysis for one period of the NLC-MDR wiggler. For comparison, we also show the corresponding analysis for the TESLA-DR. The field map we use is based on the preliminary design for the wiggler insertions discussed in [7] - at present there is no detailed design for the wiggler ends and the available field map extends only over 

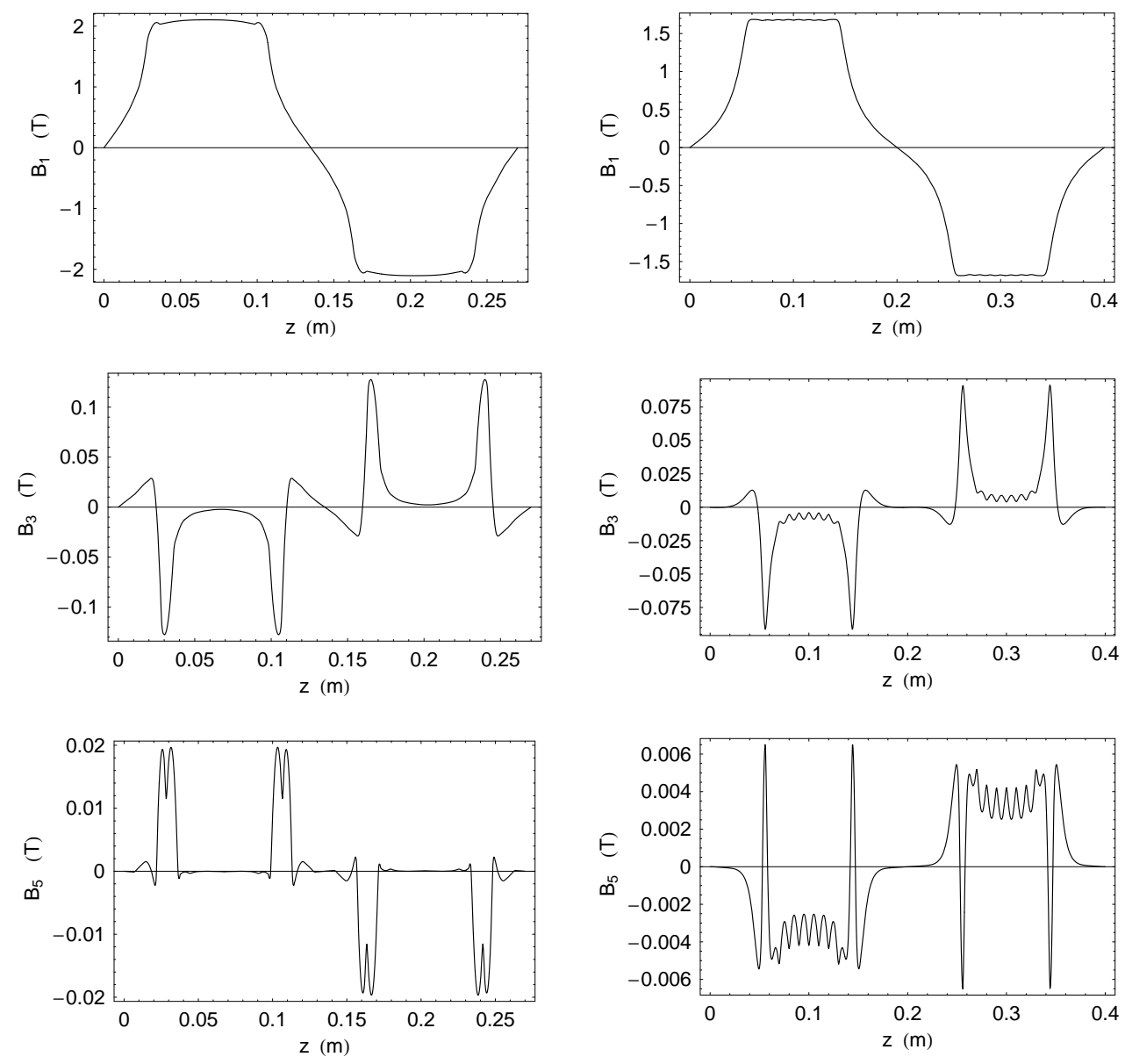

Figure 1: Field harmonics for one-period of the NLC-MDR (at $R=.9 \mathrm{~cm}$ ) and TESLA-DR (at $R=1 \mathrm{~cm}$ ) wigglers.

a single period. Compared to TESLA, the NLC damping ring wigglers have shorter period $\left(\lambda_{w}=27 \mathrm{~cm}\right.$ vs. $\left.\lambda_{w}=40 \mathrm{~cm}\right)$ and larger on-axis peak magnetic field $\left(B_{y 0}=2.1 \mathrm{~T}\right.$ vs. $\left.B_{y 0}=1.68 \mathrm{~T}\right)$. The operating energy for the NLC-MDR and TESLA-DR are $1.98 \mathrm{GeV}$ and $5 \mathrm{GeV}$ respectively. The main parameters are summarized in Table 1.

We computed the radial component of the magnetic field on the surface of a cylinder coaxial to the wigglers using the largest radius $R$ that we could fit into the available set of field data ( $R=0.9 \mathrm{~cm}$ for NLC-MDR and $R=1 \mathrm{~cm}$ for TESLA). In addition to the dominant dipole component, the Fourier analysis of $B_{\rho}$ with respect to the azimuthal angle shows that there are significant sextupole and smaller higher-order odd-harmonics. In the ideal error-free design considered here the presence of even harmonics (as well all that of the skew components) is prevented by the anti-symmetry of the fields under rotation of $180^{\circ}$ around the axis. The profiles for the odd-harmonics $B_{m}(R, z)$ through $m=5$ are plotted in Fig. 1. Their relationship to the 3D multipole expansion of $B_{\rho}$ through $5^{\text {th }}$ order in $R$ is 
Table 1: Relevant Parameters for Damping Rings and Wigglers.

\begin{tabular}{|c|c|c|}
\hline & NLC-MDR & TESLA-DR \\
\hline Energy & $1.98 \mathrm{GeV}$ & $5 \mathrm{GeV}$ \\
Rigidity $\left(q / p_{0}\right)^{-1}$ & $6.604 \mathrm{Tm}$ & $16.66 \mathrm{Tm}$ \\
Wiggler period $\lambda_{w}$ & $0.27 \mathrm{~m}$ & $0.4 \mathrm{~m}$ \\
Wiggler peak field $B_{y 0}$ & $2.1 \mathrm{~T}$ & $1.68 \mathrm{~T}$ \\
\hline
\end{tabular}

expressed by

$$
B_{\rho}=\underbrace{\left(C_{1}-\frac{3 R^{2}}{8} C_{1}^{[2]}+\frac{5 R^{4}}{192} C_{1}^{[4]}\right)}_{B_{1}(R, z)} \sin \phi+\underbrace{\left(3 C_{3} R^{2}-\frac{5 R^{4}}{16} C_{3}^{[2]}\right)}_{B_{3}(R, z)} \sin 3 \phi+\underbrace{5 C_{5} R^{4}}_{B_{5}(R, z)} \sin 5 \phi
$$

Fig. 2 shows the values of the field harmonics averaged over half-period $\left(B_{m}(R, z)\right.$ have vanishing average over one full wiggler period) and normalized with respect to the dominant $m=1$ (i.e. dipole) component. More specifically, the quantities plotted are $b_{m} / b_{1}$ with $b_{m}=$ $\int_{0}^{\lambda_{w} / 2} B_{m}(R, z) d z$. To make the comparison more meaningful in Fig. 2 the field harmonics were calculated at the same $R=0.9 \mathrm{~cm}$ for both machines.

The azimuthal harmonics of $B_{\rho}(R, \phi, z)$ evaluated on a grid of equally spaced intervals along $z$ are provided as input to a user-defined routine of MARYLIE to determine the generalized gradients and hence the vector potential through $6^{\text {th }}$. The resulting profiles of the generalized gradient $C_{1}(z)$ (equal to the on-axis verical component of the magnetic field) and some selected derivatives are shown in Fig. 3, while the generalized gradients $C_{3}(z)$ and $C_{5}(z)$ are shown in Fig. 4 . The left columns are relative to the NLC-MDR and the right column to the TESLA-DR wigglers.

In the next section we will show how these generalized gradients as they enter into the Hamiltonian through the vector potential, can be used to evaluate transfer maps.

\section{Hamiltonian and Equations of Motion for Maps}

Neglecting radiation effects the motion of a single charged particle through a general magnetic device of an accelerator can be described in a Hamiltonian framework. We choose a cartesian coordinate frame, orient the $z$-axis along the wiggler longitudinal axis, and select $z$ to be the independent "time-like" variable, while the physical time $t$ and the total energy $E$ are promoted to the status of pair of canonical coordinates $\left(t, p_{t}=-E\right)$. The corresponding Hamiltonian after a suitable scaling is

$$
H\left(x, p_{x}, y, p_{y}, t, p_{t} ; z\right)=-\sqrt{\frac{p_{t}^{2}}{p_{0}^{2} c^{2}}-\frac{m^{2} c^{2}}{p_{0}^{2}}-\left(p_{x}-\frac{q}{p_{0}} A_{x}\right)^{2}-\left(p_{y}-\frac{q}{p_{0}} A_{y}\right)^{2}}-\frac{q}{p_{0}} A_{z}
$$

where $p_{x}$ and $p_{y}$ are the transverse canonical momenta scaled with respect to the design momentum $p_{0}$. A reference orbit $\left(x^{r}, p_{x}^{r}, y^{r}, p_{y}^{r}, t^{r}, p_{t}^{r}\right)$ is a particular solution of the canonical 
NLC-MDR

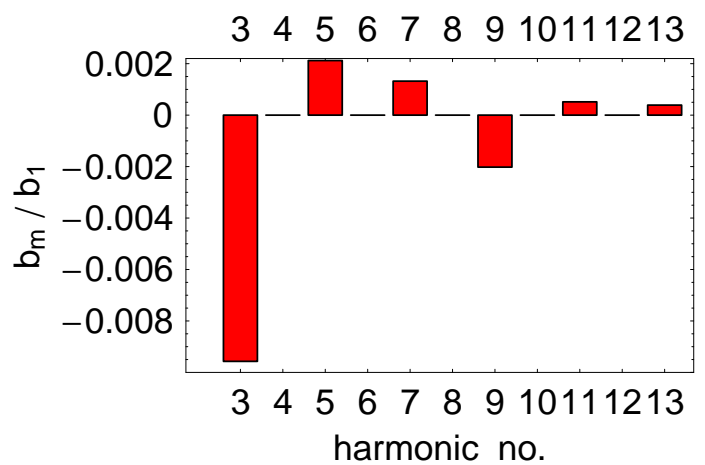

TESLA-DR

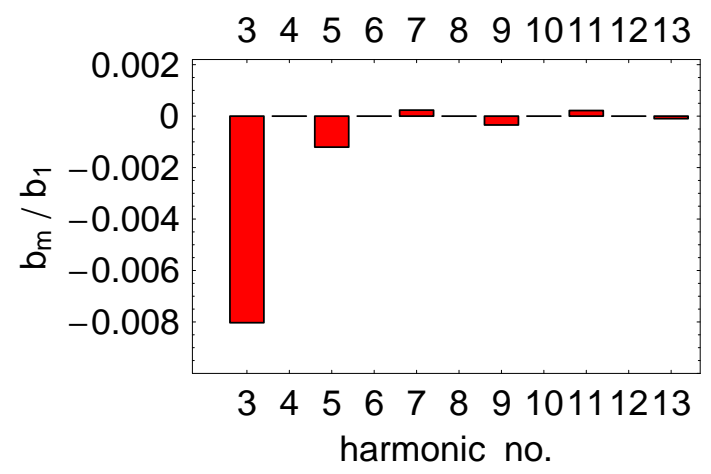

Figure 2: Field azimuthal harmonics averaged over half-period expressed in units of the leading dipole components. The dipole component is $2 b_{1} / \lambda_{w}=1.453 \mathrm{~T}$ (NLC-MDR) and $2 b_{1} / \lambda_{w}=1.020 \mathrm{~T}$ (TESLA-DR). In both cases the harmonics are evaluated at $R=0.9 \mathrm{~cm}$.

equations generated by (18). In a wiggler the reference orbit should have the same periodicity as the magnetic fields.

The non-vanishing terms of the Taylor expansion of the vector potential in cartesian coordinates for an ideal (error-free) wiggler through $6^{\text {th }}$ order

$$
\begin{aligned}
A_{x} & =x^{2} C_{1}^{[1]}-\frac{1}{8} x^{4} C_{1}^{[3]}-\frac{1}{8} x^{2} y^{2} C_{1}^{[3]}+\frac{1}{192} x^{6} C_{1}^{[5]}+\frac{1}{96} x^{4} y^{2} C_{1}^{[5]}+\frac{1}{192} x^{2} y^{4} C_{1}^{[5]}+\frac{1}{3} x^{4} C_{3}^{[1]} \\
& -x^{2} y^{2} C_{3}^{[1]}-\frac{1}{48} x^{6} C_{3}^{[3]}+\frac{1}{24} x^{4} y^{2} C_{3}^{[3]}+\frac{1}{16} x^{2} y^{4} C_{3}^{[3]}+\frac{1}{5} x^{6} C_{5}^{[1]}-2 x^{4} y^{2} C_{5}^{[1]}+x^{2} y^{4} C_{5}^{[1]}, \\
A_{y} & =x y C_{1}^{[1]}-\frac{1}{8} x^{3} y C_{1}^{[3]}-\frac{1}{8} x y^{3} C_{1}^{[3]}+\frac{1}{192} x^{5} y C_{1}^{[5]}+\frac{1}{96} x^{3} y^{3} C_{1}^{[5]}+\frac{1}{192} x y^{5} C_{1}^{[5]}+\frac{1}{3} x^{3} y C_{3}^{[1]} \\
& -x y^{3} C_{3}^{[1]}-\frac{1}{48} x^{5} y C_{3}^{[3]}+\frac{1}{24} x^{3} y^{3} C_{3}^{[3]}+\frac{1}{16} x y^{5} C_{3}^{[3]}+\frac{1}{5} x^{5} y C_{5}^{[1]}-2 x^{3} y^{3} C_{5}^{[1]}+x y^{5} C_{5}^{[1]}, \\
A_{z} & =-x C_{1}+\frac{3}{8} x^{3} C_{1}^{[2]}+\frac{3}{8} x y^{2} C_{1}^{[2]}-\frac{5}{192} x^{5} C_{1}^{[4]}-\frac{5}{96} x^{3} y^{2} C_{1}^{[4]}-\frac{5}{192} x y^{4} C_{1}^{[4]}-x^{3} C_{3} \\
& +3 x y^{2} C_{3}+\frac{5}{48} x^{5} C_{3}^{[2]}-\frac{5}{24} x^{3} y^{2} C_{3}^{[2]}-\frac{5}{16} x y^{4} C_{3}^{[2]}-x^{5} C_{5}+10 x^{3} y^{2} C_{5}-5 x y^{4} C_{5} .
\end{aligned}
$$

In studying particle orbits we are typically interested in determining the dynamics with respect to the reference orbit, that is, in terms of the deviation variables $\left(X=x-x^{r}, P_{x}=\right.$ $\left.p_{x}-p_{x}^{r}, Y=y-y^{r}, P_{y}=p_{y}-p_{y}^{r}, T=t-t^{r}, P_{t}=p_{t}-p_{t}^{r}\right)$. In these variables the Hamiltonian assumes the form

$$
\begin{aligned}
H^{d e v}=- & {\left[\left(P_{t}+p_{t}^{r}\right)^{2}-\frac{m^{2} c^{2}}{p_{0}^{2}}-\left(P_{X}+p_{x}^{r}-\frac{q}{p_{0}} A_{x}^{d e v}\right)^{2}-\left(P_{Y}+p_{y}^{r}-\frac{q}{p_{0}} A_{y}^{d e v}\right)^{2}\right]^{\frac{1}{2}} } \\
& -\left(q / p_{0}\right) A_{z}^{d e v}-\dot{x}^{r} P_{X}+\dot{p}_{x}^{r} X-\dot{y}^{r} P_{Y}+\dot{p}_{y}^{r} Y-\dot{T}^{r} P_{T}+\dot{p}_{T}^{r} T
\end{aligned}
$$



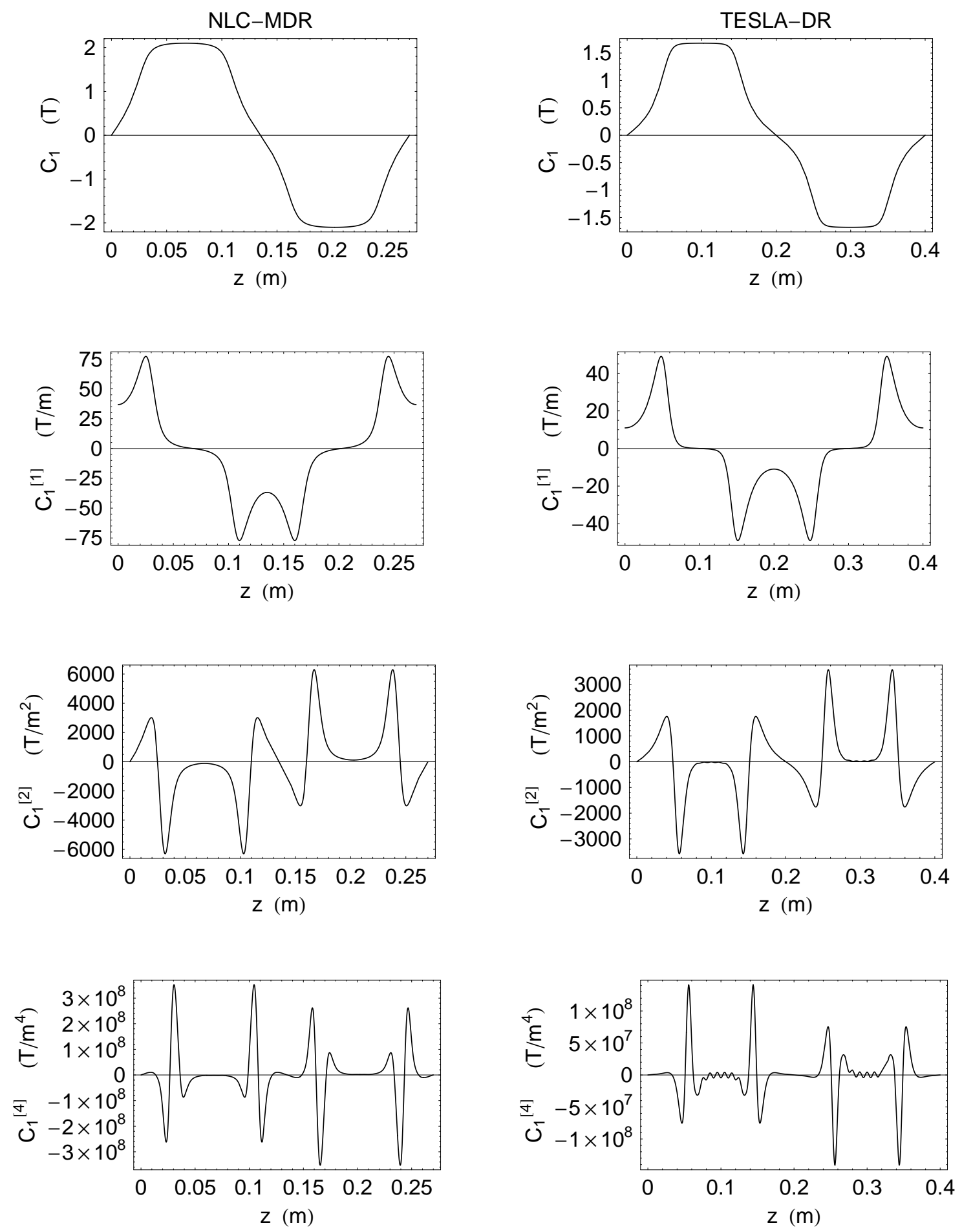

Figure 3: Generalized gradient $C_{1}(z)$ and derivatives. $C_{1}$ is equal to the the wiggler on-axis magnetic field. 

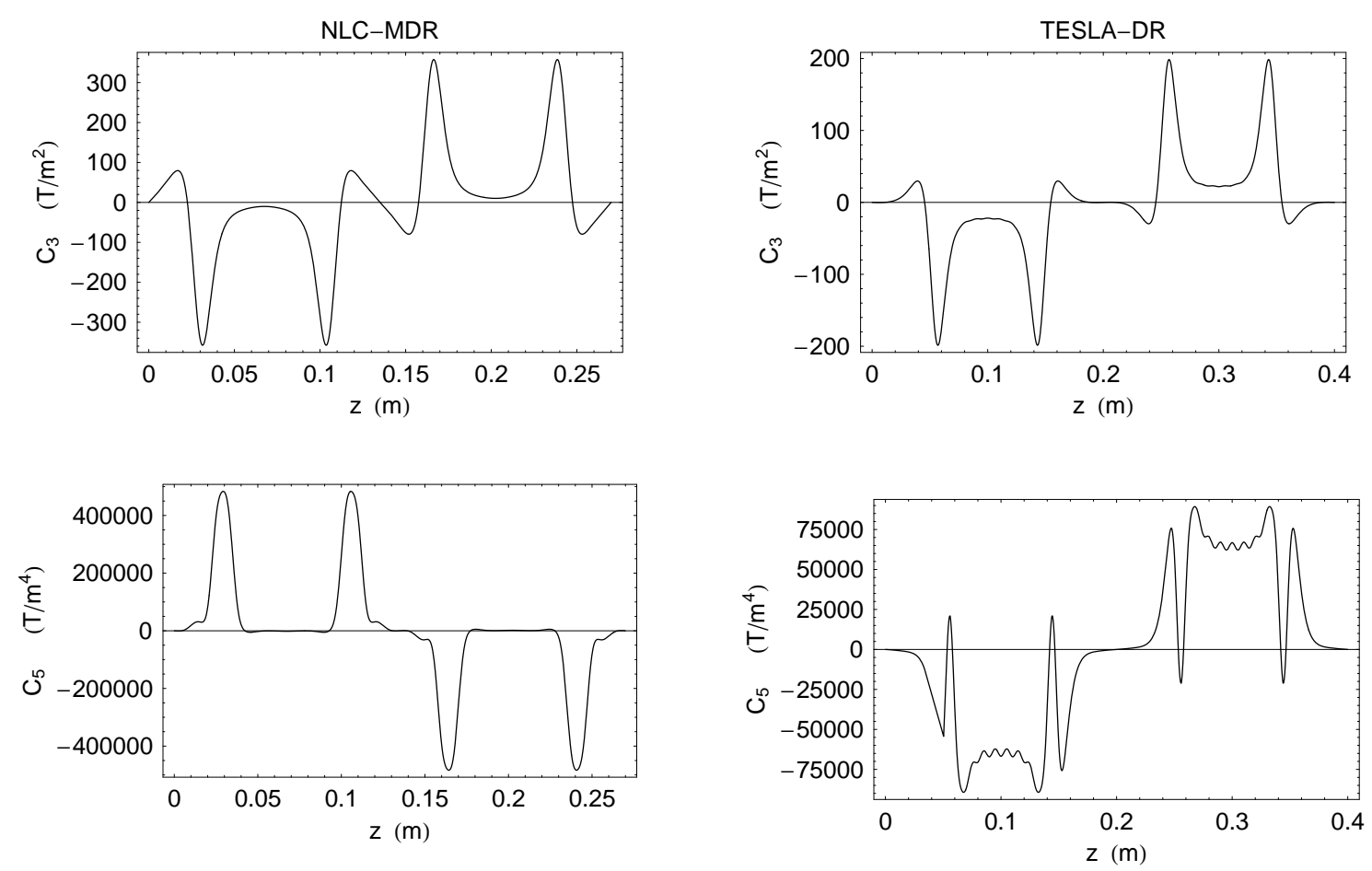

Figure 4: Generalized gradients of $3^{\text {rd }}$ (sextupole) and $5^{\text {th }}$ (decapole) order.

with the vector potential $\boldsymbol{A}^{\text {dev }}$ given by

$$
\boldsymbol{A}^{\operatorname{dev}}(X, Y, z)=\boldsymbol{A}\left(X+x^{r}, Y+y^{r}, z\right),
$$

To clarify the meaning of the transverse canonical momenta $P_{X}$ and $P_{Y}$ consider an on-momentum particle in free-space $\left(A_{x}=A_{y}=A_{z}=0\right)$. From the canonical equations of motion it follows that $d X / d z=P_{X} / \sqrt{1-P_{X}^{2}}$. By denoting with $\theta_{x}$ the angle between the tangent of the particle orbit and the $z$-axis one realizes that $P_{X}=\sin \theta_{x}$. Because $d X / d z \equiv X^{\prime}=\tan \theta_{x}$, we have $X^{\prime} \simeq P_{X}$ through second order. The same conclusion applies to $P_{Y}$.

The Hamiltonian (19) is particularly suitable for a perturbative calculation. We expand $H^{\text {dev }}=H_{2}+H_{3}+\cdots$ in a Taylor series, where the terms $H_{\ell}$ are homogeneous polynomials of degree $\ell$ in the deviation variables - by construction there is no $H_{1}$ - and solve the equations of motion for the transfer maps, order by order, from entrance to exit of a wiggler period. We do the calculation using the routine 'GENMAP' of the code MARYLIE upon specification of the generalized gradients $C_{m}^{[k]}$. The generalized gradients are evaluated from (16) at the points along $z$ required in GENMAP by the Predictor-Corrector algorithm for map integration with no interpolation needed.

In the Lie representation used by MARYLIE a symplectic transfer map describing motion around the reference orbit has the form

$$
\mathcal{M}=M e^{: f_{3}:} e^{: f_{4}: \ldots}
$$


where $M$ is the $6 \times 6$ matrix representing the linear part of the dynamics, while the $f_{\ell}$ 's are homogeneous polynomial of degree $\ell$ in the dynamical variables. The matrix $M$ depends only on $H_{2}$, the generator $f_{3}$ on $H_{3}$ and $H_{2}, f_{4}$ on $H_{4}, H_{3}$, and $H_{2}$, and so on. In version 3.0 of MARYLIE, the one employed here, the map (21) is truncated through generator $f_{4}$. For straight magnetic elements (in which the reference orbit follows the magnet axis) each individual $2 n$-pole component of the magnetic field contributes only to the $f_{\ell}$ generator with $\ell \geq n$ (the main contribution is absorbed by the generator with $\ell=n$ while the contribution to the generators with $\ell>n$ is related to the $z$-varying part of the generalized gradient - a fringe effect). However, because in wigglers the reference orbit deviates from the $z$-axis, expressing the field in the deviation coordinates (as opposed to the magnet frame coordinates) produces a cascade of feed-down terms affecting the generators with $\ell<n$ as well. For example, a sextupole component introduces a correction to the linear part of the dynamics (one can think of this term as a quadrupole-like component); a decapole component affects $f_{4}$ (octupole-like), $f_{3}$, (sextupole-like), etc. These feed-down terms are weighted by increasing powers of the deviation $x_{r}(z)$ of the reference orbit from the wiggler axis. For example, a 14-pole in the field expansion would entail a term in $A_{z}$ proportional to $x^{7}=\left(X-x^{r}\right)^{7}=-\left(x^{r}\right)^{7}+7 x\left(x^{r}\right)^{6}-21 x^{2}\left(x^{r}\right)^{5}+\cdots$. The first term on the RHS is a pure function of $z$ and has no dynamical significance. The second term would cause a correction to the reference orbit, the third term would affect $H_{2}$, the next term would modify $H_{3}$, and so on. Because for wigglers in high energy machines $x^{r}$ is fairly small $\left(x^{r}(z) \leq 0.6 \mathrm{~mm}\right.$ for the NLC-MDR), in practice for each multipole component only the first feed-down term, which is proportional to $x^{r}(z)$, is significant. For this reason not including higher order multipoles above the decapole term in the field representation (as we do) does not affect the calculation of the $3^{\text {rd }}$ order map substantially.

The transfer map obtained by integration through the fields of one wiggler period for the NLC-MDR is reported in Appendix A. In the following we will refer to it as the "GENAMP" transfer map.

The Lie form (21) is particularly efficient for map concatenation. The transfer maps through one wiggler period can be combined together to obtain those for the entire devices, and these combined with the transfer maps for the remaining lattice elements to yield the one-turn map, to be used for linear and nonlinear lattice analysis. Transfer maps for the individual elements (or collection of them) can be used to perform ray tracing as well. A numerical analysis of the transverse kicks through one wiggler period will be reported in Sec. 6 . In the following we work out some simplified analytical expressions for those kicks - useful to get a quick assessment of the dynamical role of the various terms in the field multipole expansion. To this end first we need a simplified expression for the reference orbit. The motion of the reference particle is confined to the $y=0$ plane where $A_{y}(x, y=0, z)=0$ and described by the reduced Hamiltonian $H_{x}=-\left[1-\left(p_{x}^{r}-\left(q / p_{0}\right) A_{x}\left(x_{r}, 0, z\right)\right)^{2}\right]^{\frac{1}{2}}-\left(q / p_{0}\right) A_{z}\left(x_{r}, 0, z\right)$. If we denote with $\hat{C}_{1}$ the primitive of the on-axis field $C_{1}$ that has vanishing average over one wiggler period $\int_{0}^{\lambda_{w}} d z \hat{C}_{1}=0$, the solution of the canonical equations generated by $H_{x}$ is, to the lowest order approximation

$$
x^{r}=-\frac{q}{p_{0}} \int_{0}^{z} d z^{\prime} \hat{C}_{1}\left(z^{\prime}\right), \quad p_{x}^{r}=\frac{d x^{r}}{d z}=-\frac{q}{p_{0}} \hat{C}_{1}(z)
$$



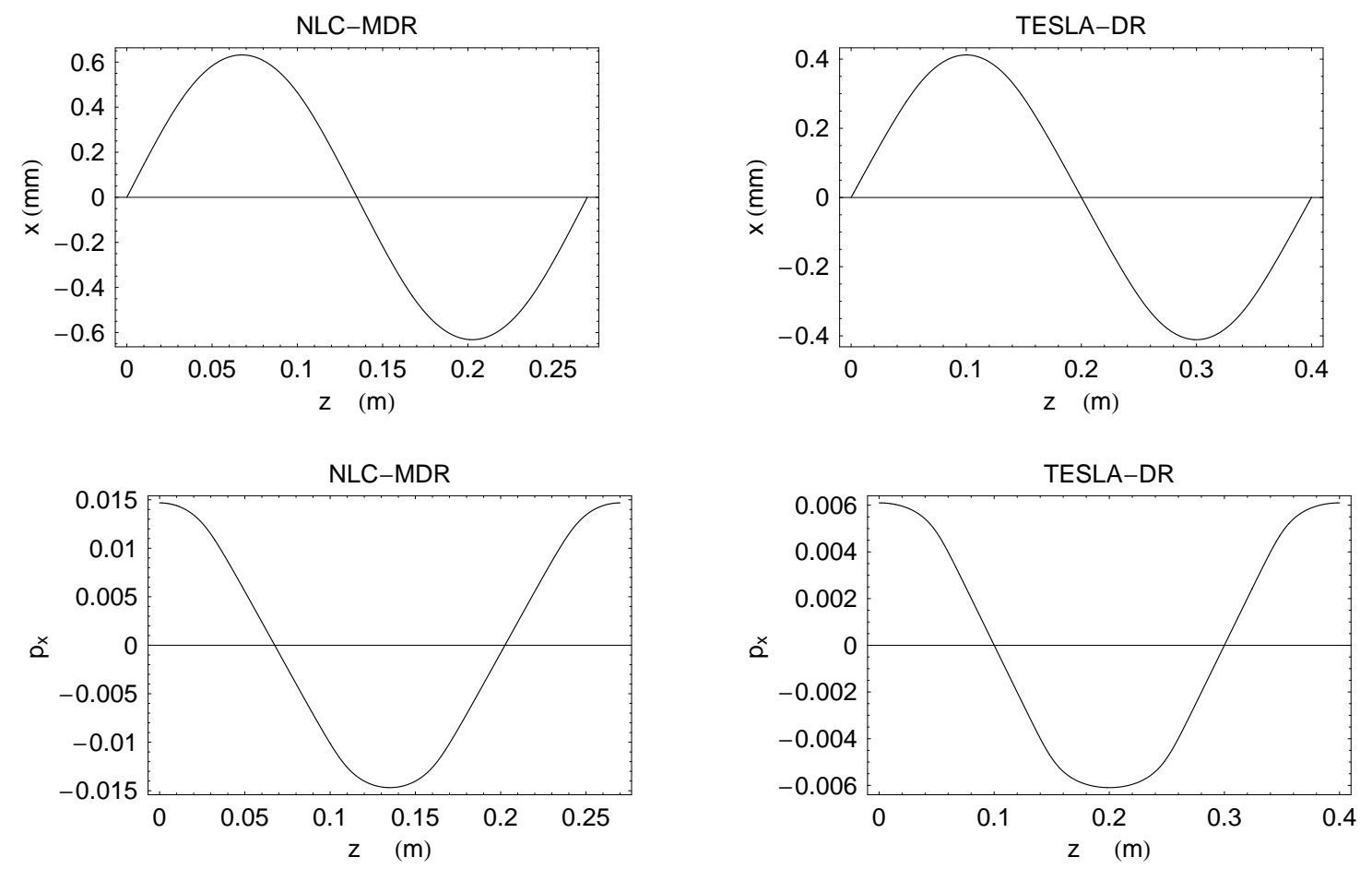

Figure 5: Reference orbits.

These expressions are fairly close to the reference orbit calculated using MARYLIE3.0. (Fig. 5). On the scale of Fig. 5 their plots would be indistinguishable from the curves shown there.

\subsection{Purely Horizontal kicks}

Consider now the dynamics in the deviation variables of an on-momentum particle with initial conditions in the horizontal plane. The reduced Hamiltonian is

$$
H_{x}=-\left[1-\left(p_{x}+p_{x}^{r}-\frac{q}{p_{0}} A_{x}^{d e v}\right)^{2}\right]^{\frac{1}{2}}-\frac{q}{p_{0}} A_{z}^{d e v}-\dot{x}^{r} p_{x}+\dot{p}_{x}^{r} x
$$

where we have changed notation and used lower case variables $x$ and $p_{x}$ in place of $X$ and $P_{X}$ to denote deviations from the reference orbit.

To evaluate the transverse kicks it is convenient to introduce a canonical transformation $\tilde{p}_{x}=p_{x}-\left(q / p_{0}\right) A_{x}^{\text {dev }}, \tilde{x}=x$ with generating function $F_{2}=x \tilde{p}_{x}+\left(q / p_{0}\right) \int A_{x}^{\text {dev }} d x$, amounting to a gauge transformation aimed at removing the $x$-component of the vector potential from under the square root in (23). The transformed Hamiltonian is given by

$$
\tilde{H}_{x}=H+\frac{\partial F_{2}}{\partial z}=-\sqrt{1-\left(\tilde{p}_{x}+p_{x}^{r}\right)^{2}}-\frac{q}{p_{0}} A_{z}^{d e v}-\dot{x}^{r}\left(\tilde{p}_{x}+\frac{q}{p_{0}} A_{x}^{d e v}\right)+\dot{p}_{x}^{r} x+\frac{q}{p_{0}} \frac{\partial}{\partial z} \int A_{x}^{d e v} d x
$$


The vector potential components $A_{x}^{d e v}$ and $A_{z}^{d e v}$, evaluated in the plane $y=0$ and through $4^{\text {th }}$ order in $x$ and first order in the reference orbit displacement $x^{r}$, read

$$
\begin{aligned}
A_{x}^{\text {dev }}= & \left(2 x x^{r}+x^{2}\right) C_{1}^{[1]}+\left(4 x^{3} x^{r}+x^{4}\right)\left(\frac{1}{3} C_{3}^{[1]}-\frac{1}{8} C_{1}^{[3]}\right), \\
A_{z}^{\text {dev }}= & -x C_{1}-\left(3 x^{2} x^{r}-x^{3}\right)\left(C_{3}-\frac{3}{8} C_{1}^{[2]}\right) \\
& -\left(5 x^{4} x^{r}-x^{5}\right)\left(C_{5}-\frac{5}{48} C_{3}^{[2]}+\frac{5}{192} C_{1}^{[4]}\right) .
\end{aligned}
$$

Under the simplifying assumption that the horizontal displacement $x$ with respect to the reference orbit remains about constant (in practice a good approximation) we obtain the transverse kick through one wiggler-period

$$
\Delta \tilde{p}_{x}=\int_{0}^{\lambda_{w}} \frac{d \tilde{p}_{x}}{d z} d z=-\int_{0}^{\lambda_{w}} \frac{\partial \tilde{H}}{\partial x} d z=\frac{q}{p_{0}} \int_{0}^{\lambda_{w}}\left(\frac{\partial A_{z, 2}^{d e v}}{\partial x}+\dot{x}^{r} \frac{\partial A_{x, 2}^{d e v}}{\partial x}\right) d z,
$$

where $A_{z, 2}^{d e v}, A_{x, 2}^{d e v}$ denote the series $(25),(26)$ with the exclusion of the terms linear in $x$ (such terms contribute only to the equations for the reference orbit - in Eq. (24) the linear parts of $-\left(q / p_{0}\right) A_{z}^{\text {dev }}$ and $-\dot{x}^{r}\left(q / p_{0}\right) A_{x}^{\text {dev }}$ are cancelled by the term $\left.\dot{p}_{x}^{r} x\right)$. The last term on the RHS of (24) does not contribute to the kick because of the periodicity of the vector potential and reference orbit $x^{r}$.

Because of the periodicity of $A_{x}^{\text {dev }}$ as a function of $z$, we recognize that the horizontal kick through one wiggler-period is the same whether expressed in terms of the old or new momentum coordinate, i.e. $\Delta \tilde{p}_{x}=\Delta p_{x}$.

Next, upon inserting expressions (25) and (26) in the above equation (27) we obtain the following estimate of linear component of and first nonlinear correction to the transverse kick

$$
\Delta p_{x}=x\left(\Delta p_{x}\right)_{1}+x^{3}\left(\Delta p_{x}\right)_{3}
$$

where

$$
\begin{aligned}
& \left(\Delta p_{x}\right)_{1}=-\frac{1}{4} \frac{q}{p_{0}} \int_{0}^{\lambda_{w}} x^{r}(z) C_{1}^{[2]}(z) d z-6 \frac{q}{p_{0}} \int_{0}^{\lambda_{w}} x^{r}(z) C_{3}(z) d z \\
& \left(\Delta p_{x}\right)_{3}=-\frac{q}{p_{0}} \int_{0}^{\lambda_{w}} x^{r}(z)\left(\frac{C_{1}^{[4]}(z)}{48}-\frac{3 C_{3}^{[2]}(z)}{4}+20 C_{5}(z)\right) d z .
\end{aligned}
$$

In (25) and (26) the terms not proportional to the displacement $x^{r}$ of the reference orbit average to zero and in the present approximation do not contribute to the kicks. By integrating by parts twice and making use of (22) the first term on the RHS of Eq. (29) can be rewritten as $-\left(q / p_{0}\right)^{2} \int_{0}^{\lambda_{w}} C_{1}^{2}(z) d z / 4$. This term is always present ${ }^{\S}$ and carries a negative

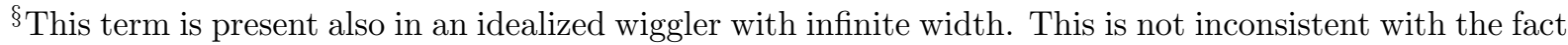
that in this case there is no horizontal focusing, as feed-downs from higher order multipoles (see Appendix C) will compensate the focusing effect of $C_{1}(z)$, causing $\left(\Delta p_{x}\right)_{1}=0,\left(\Delta p_{x}\right)_{3}=0$, etc.
} 
Table 2: Transverse Kicks for One Wiggler Period

\begin{tabular}{|l|l|l|}
\hline & NLC-MDR & TESLA-DR \\
\hline$\left(\Delta p_{x}\right)_{1 a}=-\left(q / p_{0}\right)^{2} \int_{0}^{\lambda_{w}} C_{1}^{2} d z / 4$ & $-0.00402 \mathrm{~m}^{-1}$ & $-0.00052 \mathrm{~m}^{-1}$ \\
$\left(\Delta p_{x}\right)_{1 b}=-6\left(q / p_{0}\right) \int_{0}^{\lambda_{w}} x^{r} C_{3} d z$ & 0.00740 & 0.00172 \\
$\left(\Delta p_{x}\right)_{1}=\left(\Delta p_{x}\right)_{1 a}+\left(\Delta p_{x}\right)_{1 b}$ & 0.00337 & 0.00120 \\
$\left(\Delta p_{y}\right)_{1}$ & -0.0194 & -0.00329 \\
\hline$\left(\Delta p_{x}\right)_{3 a}=-\left(q / p_{0}\right)^{2} \int_{0}^{\lambda_{w}} x^{r} C_{1}^{[4]} d z / 48$ & $-0.214 \mathrm{~m}^{-3}$ & $-0.016 \mathrm{~m}^{-3}$ \\
$\left(\Delta p_{x}\right)_{3 b}=3\left(q / p_{0}\right) \int_{0}^{\lambda_{w}} x^{r} C_{3}^{[2]} d z / 4$ & 0.561 & 0.060 \\
$\left(\Delta p_{x}\right)_{3 c}=-20\left(q / p_{0}\right) \int_{0}^{\lambda_{w}} x^{r} C_{5} d z$ & -30.02 & 6.31 \\
$\left(\Delta p_{x}\right)_{3}=\left(\Delta p_{x}\right)_{3 a}+\left(\Delta p_{x}\right)_{3 b}+\left(\Delta p_{x}\right)_{3 c}$ & -29.68 & 6.35 \\
$\left(\Delta p_{y}\right)_{3}$ & -32.03 & 6.13 \\
\hline
\end{tabular}

sign, indicating horizontal focusing. The second term on the RHS of Eq. (29) is a feed-down from the azimuthal sextupole field component. The overall sign will depend on the relative sign of and ratio between $C_{1}$ and $C_{3}$. From Table 2 we can see that the sign of $\left(\Delta p_{x}\right)_{1}$ is positive in both the NLC-MDR and TESLA-DR wigglers. Feed-down from higher order azimuthal harmonics would also contribute to linear focusing but their relative contributions are of order $\left(x^{r}\right)^{2}$ or higher and are neglected here. As for the nonlinear correction $\left(\Delta p_{x}\right)_{3}$, Table 2 indicates that by and large the dominant component is that involving $C_{5}$ (decapole feed-down).

\subsection{Purely Vertical Kicks}

Similarly, way can evaluate vertical kicks for a particle entering a wiggler period in the $x=0$ plane with a vertical offset $y$ by use of the reduced Hamiltonian

$$
H_{y}=-\left[1-\left(p_{y}-\frac{q}{p_{0}} A_{y}^{d e v}\right)^{2}\right]^{\frac{1}{2}}-\frac{q}{p_{0}} A_{z}^{d e v},
$$

Once again, we remove the transverse component of the vector potential from under the square root in (31) by a canonical transformation $\tilde{p}_{y}=p_{y}-\left(q / p_{0}\right) A_{y}^{\text {dev }}, \tilde{y}=y$ with generating function $F_{2}=y \tilde{p}_{y}+\left(q / p_{0}\right) \int A_{y}^{d e v} d y$. The transformed Hamiltonian reads

$$
\tilde{H}_{y}=H_{y}+\frac{\partial F_{2}}{\partial z}=-\sqrt{1-\tilde{p}_{y}^{2}}-\frac{q}{p_{0}} A_{z}^{d e v}+\frac{q}{p_{0}} \frac{\partial}{\partial z} \int A_{y}^{d e v} d x .
$$

Assuming that the horizontal displacement $y$ with respect to the reference orbit remains constant the transverse kick reads

$$
\Delta \tilde{p}_{y}=\int_{0}^{\lambda_{w}} \frac{d \tilde{p}_{y}}{d z} d z=\frac{q}{p_{0}} \int_{0}^{\lambda_{w}} \frac{\partial A_{z}^{d e v}}{\partial y} d z .
$$

As no dependance on the vertical component $A_{y}^{\text {dev }}$ is left in (33) after integration only the expression for $A_{z}^{\text {dev }}$ need be reported. Through $4^{t h}$ order in $y$ and first order in the reference orbit displacement $x^{r}$ it is 


$$
A_{z}^{\text {dev }}=3 y^{2} x^{r}\left(C_{3}+\frac{3}{8} C_{1}^{[2]}\right)-5 y^{4} x^{r}\left(C_{5}+\frac{1}{16} C_{3}^{[2]}+\frac{1}{192} C_{1}^{[4]}\right) .
$$

As before, the vertical kick is the same whether expressed in the old or new variables, $\Delta \tilde{p}_{y}=\Delta p_{y}$. Specifically

$$
\Delta p_{y}=y\left(\Delta p_{y}\right)_{1}+y^{3}\left(\Delta p_{y}\right)_{3},
$$

with

$$
\begin{aligned}
& \left(\Delta p_{y}\right)_{1}=-\frac{3}{4} \frac{q}{p_{0}} \int_{0}^{\lambda_{w}} x^{r}(z) C_{1}^{[2]}(z) d z+6 \frac{q}{p_{0}} \int_{0}^{\lambda_{w}} x^{r}(z) C_{3}(z) d z \\
& \left(\Delta p_{y}\right)_{3}=-\frac{q}{p_{0}} \int_{0}^{\lambda_{w}} x^{r}(z)\left(\frac{5 C_{1}^{[4]}(z)}{48}+\frac{5 C_{3}^{[2]}(z)}{4}+20 C_{5}(z)\right) d z .
\end{aligned}
$$

In the absence of higher order azimuthal harmonics the coefficient of the linear part of the vertical kick is negative i.e. a planar wiggler naturally provides focusing in both planes (similarly to (29) the first term on the RHS of (36) can be written as $\left.-3\left(q / p_{0}\right)^{2} \int_{0}^{\lambda_{w}} C_{1}^{2}(z) d z / 4\right)$. Also, notice that the strength of this dipole-component induced focusing in the vertical plane [first term on the RHS of (36)] is three times as large as in the horizontal plane.

\section{Connection with the 'Dynamical Field Integral'}

From Eq. (38) the expressions for the magnetic field through $4^{\text {th }}$ order in cartesian coordinates $B_{x}=B_{\rho} \cos \phi-B_{\phi} \sin \phi, B_{y}=B_{\rho} \sin \phi+B_{\phi} \cos \phi$ read:

$$
\begin{aligned}
B_{x} & =-\left(\frac{1}{4} C_{1}^{[2]}-6 C_{3}\right) x y+\left(\frac{1}{48} C_{1}^{[4]}-\frac{3}{4} C_{3}^{[2]}+20 C_{5}\right) x^{3} y+\left(\frac{1}{48} C_{1}^{[4]}-\frac{1}{4} C_{3}^{[2]}-20 C_{5}\right) x y^{3}, \\
B_{y} & =C_{1}-\left(\frac{1}{8} C_{1}^{[2]}-3 C_{3}\right) x^{2}-\left(\frac{3}{8} C_{1}^{[2]}+3 C_{3}\right) y^{2}+\left(\frac{1}{192} C_{1}^{[4]}-\frac{3}{16} C_{3}^{[2]}+5 C_{5}\right) x^{4}, \\
& +\left(\frac{1}{32} C_{1}^{[4]}-\frac{3}{8} C_{3}^{[2]}-30 C_{5}\right) x^{2} y^{2}+\left(\frac{5}{192} C_{1}^{[4]}-\frac{5}{16} C_{3}^{[2]}+5 C_{5}\right) y^{4} \\
B_{z} & =y C_{1}^{[1]}-\left(\frac{1}{8} C_{1}^{[3]}-3 C_{3}^{[1]}\right) x^{2} y-\left(\frac{1}{8} C_{1}^{[3]}-C_{3}^{[1]}\right) y^{3} .
\end{aligned}
$$

The expression for $B_{y}$ can be used to determine the 'dynamical field integral' introduced and discussed in Ref. [9], which reports on the experience gained from the wiggler insertion BL11 in the SPEAR ring at SLAC. Because of poor design the nonlinearities introduced by this insertion were found to cause unacceptable degradation of the beam lifetime. A successful correction strategy was devised that required placing a combination of quadrupole, octupole, and dodecapole magnet blocks ('magic fingers') at the two wiggler ends to compensate for both the linear and nonlinear perturbation to the dynamics. The strength of the multipoles was set as to minimize the vertical magnetic field integrated over a particle orbit $x(z)$ in the wiggler midplane i.e. $I_{y}=\int B_{y}(x(z), y=0, z) d z$ (the 'dynamical field integral'). 
If one assumes that the particle orbit can be written as $x=x^{r}(z)+X$, where $x^{r}$ is the reference orbit and $X$ remains constant across the wiggler, the quantity $I_{y}$ turns out to be proportional to the horizontal kick experienced by the particle. Not surprisingly, as this is the same assumption that led to our simplified calculation of Sec. 5.1 one recovers through order $\mathcal{O}\left(x^{r}\right)$ an expression similar to Eq. (28)

$$
\begin{aligned}
I_{y} & =X \int_{0}^{L_{w}} x^{r}(z)\left(-\frac{1}{4} C_{1}^{[2]}(z) d z+6 x^{r}(z) C_{3}(z) d z\right) \\
& +X^{3} \int_{0}^{L_{w}} x^{r}(z)\left(\frac{C_{1}^{[4]}(z)}{48}-\frac{3 C_{3}^{[2]}(z)}{4}+20 C_{5}(z)\right) d z .
\end{aligned}
$$

In [9] it was correctly pointed out that minimizing $I_{y}$ does not necessarily cause an overall compensation of the wiggler perturbation to the beam dynamics. However, we should observe that it may nevertheless result in softer nonlinearities in the vertical plane as well. Indeed, this would be the case to the extent that the assumption leading to the simplified calculation of Sec. 5.1 and 5.2 hold and the term in (39) proportional to $C_{5}$ dominates over those containing derivatives of the generalized gradients. Inspection of Eq.(37) shows that for the vertical kick the term with $C_{5}$ would also be dominant and automatically compensated by an octupole magnet tuned to correct the horizontal nonlinearity.

The same considerations would presumably also apply to a $X^{5}$ nonlinearity (not shown in Eq. (39)) dominated by the term $C_{7}$ that would result from a 14-pole in the wiggler magnetic field expansion. Correcting this nonlinearity would require a dodecapole magnet. It is in our plans to investigate this point in the future by applying our analysis to a detailed field map of the BL11 insertion.

\section{$7 \quad$ Evaluation of the Transverse Kicks by Transfer Maps}

In MARYLiE tracking through a lattice element or lump of lattice elements can be carried out using two options. The first is to express map (21) as a truncated Taylor series in the dynamical variables (through $3^{\text {rd }}$ order in version 3.0 of MARYLIE). In general this truncation is nonsymplectic. Alternatively, one can chose a second option amounting to completing the truncated Taylor series with fictitious high order terms in such a way that the completion is symplectic. There is a general consensus that long term tracking should be done using a symplectic integrator, however, for single pass calculations, there is little difference in the outcome between the two options (at least if the orbit amplitude in phase space is not extreme).

Here we report the result of calculating the transverse kicks using the map through one wiggler-period and the symplectic option for ray-tracing. The particles have initial vanishing transverse canonical momenta. The horizontal (vertical) kicks are evaluated by

\footnotetext{
๑From [9], however, it is not clear whether the correction scheme adopted for the SPEAR BL11 insertion while proving itself very effective in ameliorating the dynamics in the horizontal plane (the more critical for machine performance) was found to be beneficial for the vertical motion as well.
} 

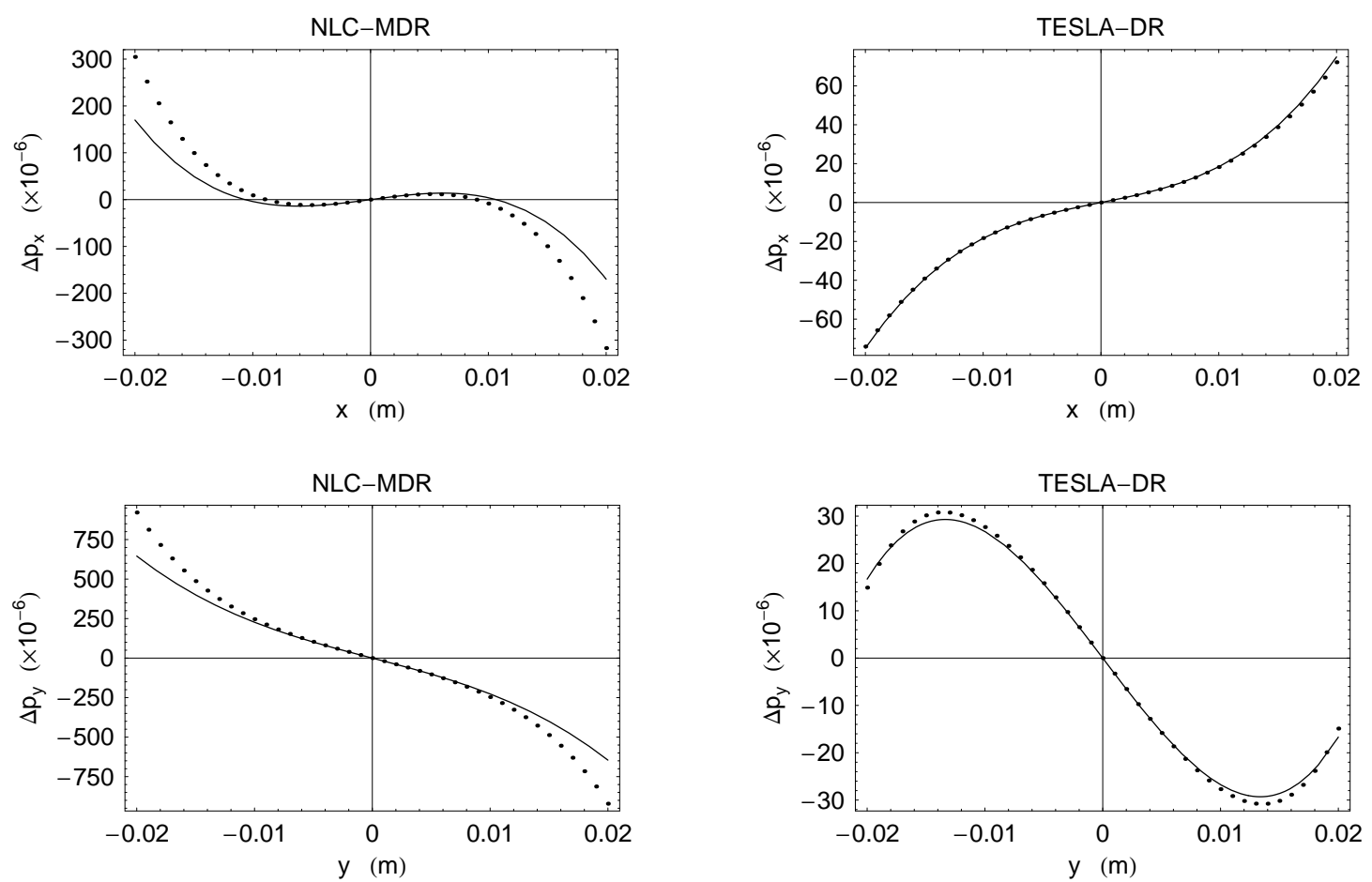

Figure 6: Horizontal (top row) and vertical kicks (bottom row) through one wiggler period. Solid lines are from the simplified analytical expressions of Sec. 5.1 and 5.2. Dots are from the MARYLiE map calculation.

selecting initial positions in the horizonal $y=0$ (vertical $x=0$ ) plane. The results of the MARYLIE calculation are shown in Fig. 6 as dots. The solid lines represent an evaluation of the transverse kicks according the simplified expressions of Sec. 5.1 and 5.2. As expected, for small amplitudes, the outcomes from the MARYLIE calculation and the simplified expressions agree well. However, in the case of the NLC-MDR wiggler which suffers relatively larger kicks the agreement is spoiled at larger amplitudes. As the field quality in the wigglers for the two machines is comparable (see Fig. 2 the difference in the amplitude of the kicks is mostly due to the difference in the rigidity (see Table 1). We recall that transverse kicks in wigglers scale with the inverse of the square of the rigidity. This is in contrast to the scaling of the transverse kicks in ordinary lattice elements, which are proportional to only the inverse of the rigidity.

\section{Modelling of one Wiggler Period Using Standard El- ements}

The values reported in Table 2 suggest that the leading nonlinearity in both the horizontal and vertical planes come from feed-downs of the decapole term in the $A_{z}$ component of the vector potential (terms proportional to $C_{5}$ ). In contrast, feed-down contributions propor- 


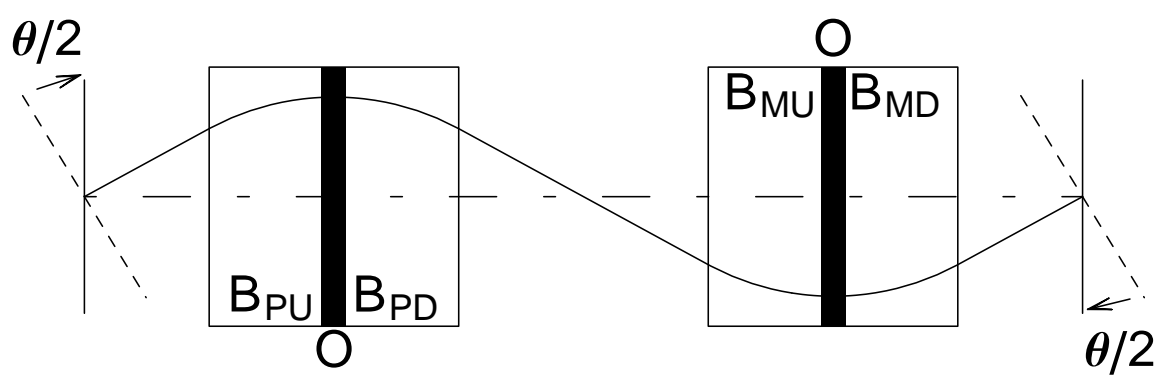

Figure 7: Outline of the sequence of standard elements with bends and thin octupoles (black bands) corresponding to (41). The reference orbit is indicated (sinusoidal-like curve) as well as the transverse plane rotation by $\theta / 2$ at the two ends where $\theta$ is the bending angle through a wiggler pole (see remark at the end of this Section).

tional to derivatives of $C_{1}(z)$ and $C_{3}(z)$, which appear in the $A_{x}$ and $A_{y}$ components of the vector potential are relatively small. To the extent that in the $A_{\phi}=0$ gauge, which we have used throughout, the contribution to the dynamics from $A_{x}$ and $A_{y}$ can be neglected and particle orbits do not deviate considerably from straight lines in the deviation variables - we can conclude that through $\mathcal{O}\left(x^{r}\right)$ and $3^{r d}$-order the wiggler nonlinearities would have the same form as that of a standard octupole magnet. This can be easily seen: as the $A_{z}$-component of a decapole in the wiggler coordinate frame reads $A_{z}=-C_{5}\left(x^{5}-10 x^{3} y^{2}+5 x y^{4}\right)$, in terms of the deviation variables $X=x-x^{r}, Y=y$ we have

$$
\begin{aligned}
A_{z} & =-C_{5}\left(\left(X+x^{r}\right)^{5}-10\left(X+x^{r}\right)^{3} Y^{2}+5\left(X+x^{r}\right) Y^{4}\right) \\
& \equiv-5 C_{5} x^{r}\left(X^{4}-6 X^{2} Y^{2}+Y^{4}\right)+\mathcal{O}\left(\left(x^{r}\right)^{2}\right)
\end{aligned}
$$

As anticipated, the expression appearing the RHS has the same form as that of a standard octupole field component. In the last line of the above equation we omitted the terms without a dependence on $x^{r}$, as they integrate to zero and in first approximation do not contribute to the dynamics.

On the basis of these observations it seems reasonable to try to model the map of a wiggler period by a suitable combination of standard elements, with inclusion of (thin) octupoles to model the $3^{r d}$-order nonlinearities. In particular, we have considered the sequence (see also Fig. 7)

$$
D_{\lambda_{w} / 8} B_{P U} O B_{P D} D_{\lambda_{w} / 4} B_{M U} O B_{M D} D_{\lambda_{w} / 8}
$$

where $D_{\lambda_{w} / 8}, D_{\lambda_{w} / 4}$ represent drifts of length $\lambda_{w} / 8$ and $\lambda_{w} / 4\left(\lambda_{w}\right.$ is the length of the wiggler period). $B_{P U}$ and $B_{P D}$ are combined-function bends with positive polarity, each one modelling half a wiggler pole; $B_{M U}$ and $B_{M D}$ are combined-function bends with negative polarity. Each half-pole has length $\lambda_{w} / 8$. Two thin octupoles $O$ are placed between the half-bends.

While the dipole field, $B_{y 0}=2.277 \mathrm{~T}$, is determined by the requirement set to achieve the desired damping rate in the ring there remain two free parameters: the strength of the 

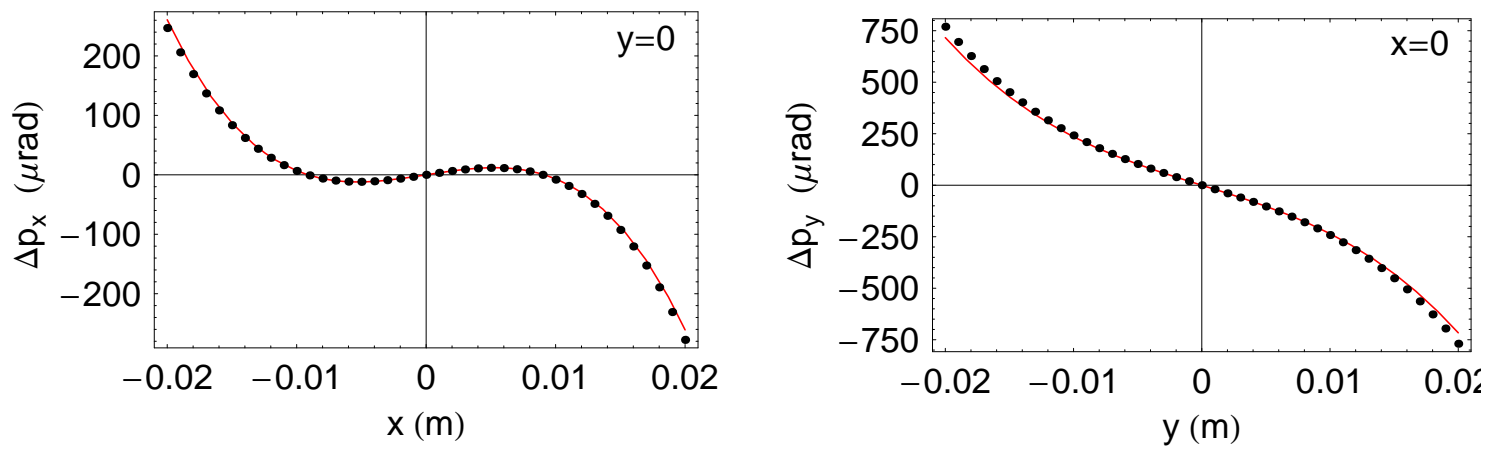

Figure 8: Horizontal (left) and vertical kicks (right) through one wiggler period calculated by integration with GENMAP (dots) and using the standard element model (line). Particles at entry have vanishing transverse momenta. These kicks are "in-plane", that is $y=0$, when calculating the horizontal kick, and $x=0$ when calculating the vertical kick.

quadrupole component in the combined function bends and the strength of the octupole thin lenses. We use these free parameters to force the transfer map corresponding to the sequence (41) to be close in some way to the wiggler one-period transfer map that we computed by integrating through the actual fields (see Appendix A).

We used the first parameter to fit the elements $M_{43}$ of the linear part of the map and the second to fit the : $x^{4}$ : Lie generator against the corresponding matrix element and Lie generator of the accurate map. The resulting field gradient in the combined function bend is found to be $-0.1665 \mathrm{~T} / \mathrm{m}$ (defocusing in the horizontal plane), the strength of each of the two thin octupoles $134.79 \mathrm{~T} / \mathrm{m}^{2}$ (corresponding to $\mathrm{K} 3 \mathrm{~L}=122.45 \mathrm{~m}^{-3}$ in MAD notation).॥

The map resulting from the fit is reported in Appendix B - comparison with the GENMAP calculation (Appendix A) shows a reasonable agreement for its linear part. All the corresponding matrix entries are within few percent or less, except for the entry $M_{16}$ (and $M_{61}$ ) which shows a deviation of the order of $20 \%$ but is, however, very small. As for the nonlinear generators some happen to be relatively close like the $: y^{4}:$ generator (off by about $17 \%)$ while others, like the generator $: x^{2} y^{2}$, differ by a factor 2 or larger. Incidentally, the first of these two generators dominates the nonlinear kick in the horizontal plane while the second contributes to the $x$ - $y$ coupling. However, it is difficult to assess the dynamical effects of the two maps just by inspection of the Lie-generators. More revealing is a comparison between the kicks obtained by application of the two maps, and ultimately, evaluation of their impact on the DA of the ring lattice. We will present the DA study later in Sec. 10. Here we show a graphical comparison (Figs. 8 and 9) between the kicks relative to one wiggler period evaluated using the two different maps. The pictures show a reasonable good agreement except for the off-plane vertical kicks with a finite horizontal displacement (picture to the right in Fig. 9).

We end this Section with a technical remark. The map calculated using GENMAP

${ }$ The MAD notation $K_{n}$ for a multipole of order $n$ ( $n=1$ quadrupole, $n=2$ sextupole, etc.) is related to the MARYLIE notation $M_{n}$ by $M_{n}=K_{n} B r h o / n$ !, where Brho is the rigidity. 

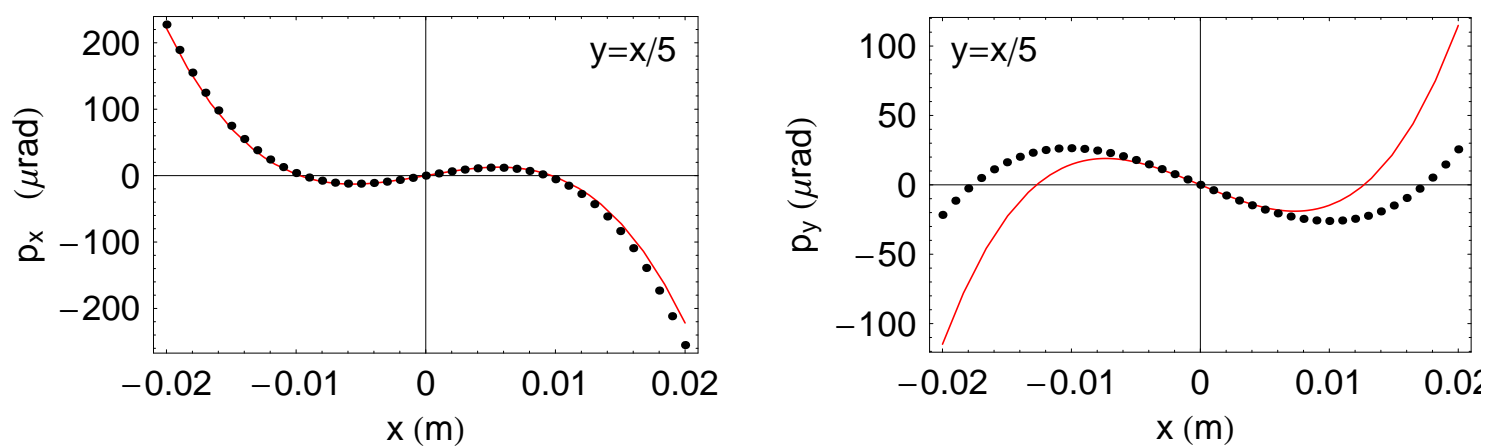

Figure 9: Horizontal (left) and vertical kicks (right) through one wiggler period calculated by integration with GENMAP (dots) and using the standard element model (line). Particles at entry have vanishing transverse momenta. Kicks are "off-plane": in both case the horizontal $(x)$ and vertical $(y)$ displacement of the particles at entry are related by $y=x / 5$.

presupposes that the initial and final positions of the particle orbits lie on planes perpendicular to the $z$-axis (the wiggler longitudinal axis). In contrast, when combining the maps indicated by the sequence (41) it is understood that initial and final positions of the particle orbits lie on a plane placed at an angle $\theta / 2$ with respect to the wiggler axis (dashed lines in Fig. 7), where $\theta$ is the bending angle through one wiggler pole. In order to make a more meaningful comparison one should precede and follow the application of the map (41) by suitable rotations of the transverse plane. Such an action in MARYLIE is carried out by the command 'prot'. Both these rotations were accounted for in the calculation leading to the map reported in Appendix B and used to produce the pictures shown in this Section.

\section{Compensating the Wiggler $3^{r d}$ Order Nonlinearities with Octupole Correctors.}

Depending on the particular application and wiggler design it may be desirable or necessary to correct for the nonlinear perturbations added to the beam dynamics. As already mentioned in Sec. 6 this was the case for the 1998 installation of the BL11 insertion in SPEAR. The adopted correction scheme consisted in placing two sets of magnets in a quadrupole, octupole, and dodecapole configuration aimed at cancelling the 'dynamical field integral' shown in Sec 6. The results from the previous Section suggest an alternative and straightforward strategy. Suppose we are interested in correcting the $3^{r d}$-order nonlinearities - the linear perturbation in principle can always be taken care of by adjusting the linear lattice in the rest of the machine. Having calculated the map obtained by integrating through the realistic wiggler fields (Sec. 5) and its best fit in terms of a sequence of standard magnets (Sec. 8), one can set up octupole correctors that have strength opposite of those used to construct the fitting map. Ideally, one would like to place the correctors in correspondence of each wiggler poles. However, in a more realistic setting the correctors should be placed at the insertion ends - at the cost of partially spoiling the compensation because of the particle 

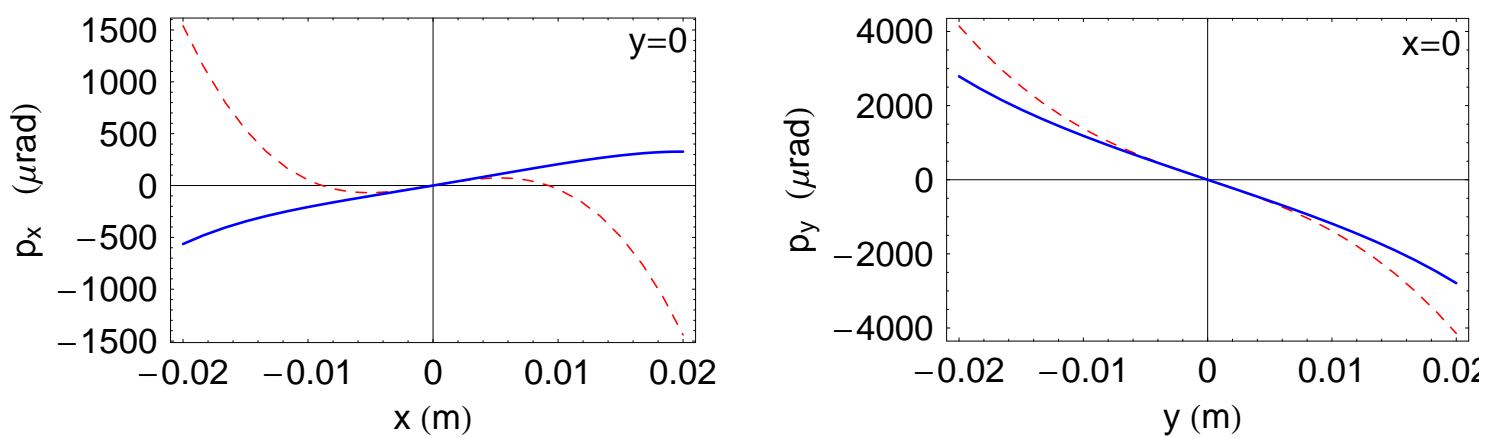

Figure 10: Horizontal (left) and vertical (right) kicks through 6 wiggler periods with (solid lines) and without (dashed line) octupole magnet correctors.

finite phase advance.

We tested the correction scheme by comparing the total horizontal and vertical kicks obtained by 6 repeated applications of the one-period map and the kicks obtained by preceding and following the same sequence with the application of the maps of two thin octupoles. This sequence corresponds roughly to the body of the wiggler insertions to be used in the MDR lattice. In this discussion we ignore the effects due to the insertion ends. The strength of each of the two thin octupoles was set to be $-6 \times 2 \times 134.79 / 2=-808.74 \mathrm{~T} / \mathrm{m}^{2}$, a value resulting from the condition that the sum of the strength of the two correctors be opposite of the integrated strength of the octupoles in the one-period fitting map discussed in Sec. 8 . Fig. 10 shows the horizontal and vertical kicks through 6 full wiggler periods with and without correctors. The effectiveness of the correction is apparent form the improved linearity of the shape of the curves. In the horizontal plane one can observe the presence of some residual quadratic terms (sextupolar-like). The fact that the correction of the nonlinearities seems to be slightly less effective in the vertical plane (inspection of the right picture in Fig. 10 shows a residual curvature in the line representing the kick) appears to depend to both the fact that the correction was targeted to the horizontal plane** and that the perturbation to the linear motion is stronger (and the resulting phase advance larger) in the vertical than in the horizontal plane.

In the same way one can conceivably think of correcting $5^{\text {th }}$-order (and possibly higher) nonlinearities provided that the main contribution to the corresponding kicks be caused by feed-downs from the 14-pole term in the multipole expansion of $A_{z}$ (proportional to the generalized gradient $C_{7}$ ). We plan to investigate this point in the future by using version 5.0 of MARYLIE.

Notice that a difference in the absolute values of linear focusing (in $y$ ) and defocusing (in $x$ ) prevents simultaneous compensation of the linear part of the dynamics in both planes by means of additional quadrupole magnet correctors. This is related to the fact that in Eqs. (29) and (36) the terms to the left are not negligible compared to the terms on the

${ }^{* *}$ Recall that in Sec. 8 fitting of the thin octupoles in the sequence of standard elements model for the wiggler period was done by selecting the Lie generator $: x^{4}$ :. 
Table 3: Quadrupole setting for the matching of the wiggler insertions into the MDR lattice. Quadrupole strength is K1 (MAD notation). Values in brackets indicate the corresponding gradient.

\begin{tabular}{|c|c|c|}
\hline & Feb. 03 Design $\left(\mathrm{m}^{-2}\right)$ & Present Study $\left(\mathrm{m}^{-2}\right)$ \\
\hline QDW & -1.21060 & $-0.79739[-5.26646 \mathrm{~T} / \mathrm{m}]$ \\
QFW & 3.21384 & $2.60961[17.23533 \mathrm{~T} / \mathrm{m}]$ \\
Q1WM & 4.08070 & $4.55004[30.05107 \mathrm{~T} / \mathrm{m}]$ \\
Q2WM & -4.65844 & $-4.85085[-32.0378 \mathrm{~T} / \mathrm{m}]$ \\
Q3WM & 3.17147 & $3.89201[25.7051 \mathrm{~T} / \mathrm{m}]$ \\
Q4WM & -1.21710 & $-1.16545[-7.69728 \mathrm{~T} / \mathrm{m}]$ \\
\hline
\end{tabular}

right. Only the latter terms, coming from feed-downs in the sextupole components in the $A_{z}$-field expansion, display the azimuthal symmetry of the fields of a quadrupole magnet.

Finally we should point out that the tuning of any correction scheme based on the use of standard multipole magnets is strictly dependent on the beam energy because - as already pointed out - the wiggler nonlinearities scale with the inverse of the square of rigidity while the kicks from standard magnet elements scale only with its inverse.

\section{Dynamic Aperture for the NLC-MDR (Error-Free) Lattice with Wigglers}

The present NLC-MDR lattice design [1] includes 32 wiggler insertions equally divided between the ring two main straight sections and occupying about $62 \mathrm{~m}$ of the $300 \mathrm{~m}$ machine circumference. Each wiggler insertion consists of 14 equally separated full poles and two half-poles at the ends. The field configuration is such that a particle entering the insertion on axis remains on axis at the exit. In [1] the wigglers were modelled using the same sequence of standard magnet elements (combined function dipoles, octupoles) that we considered in Sec. 8 but with a different choice for the magnet parameters. In the present study we used the same lattice as in [1] with two exceptions: first of all, we replaced the standard elements in the wiggler insertions spanning one wiggler period (two poles) with the one-period transfer map calculated by integrating through a realistic representation of the fields (see Sec. 5). As a complete design for the wiggler insertions including the ends and the corresponding field map is unfortunately not available yet, we maintained a representation of the wiggler ends in terms of standard elements (with the magnet parameters set as in Sec. 8). Each insertion end consists of one half-pole and one full pole: the half-pole was modelled as a simple combined function dipole; and the contiguous full pole (half-period) as a combined function dipole with a thin sextupole in addition to a thin octupole. ${ }^{\dagger \dagger}$ The need of an additional thin sextupole results from the observation that over half a full wiggler period the contribution

\footnotetext{
${ }^{\dagger \dagger}$ In summary, of the 7 full periods in each wiggler insertion 6 have been modelled using the accurate maps of Sec. 5 and the remaining one (split into two halves placed at the two insertion ends) using standard elements.
} 


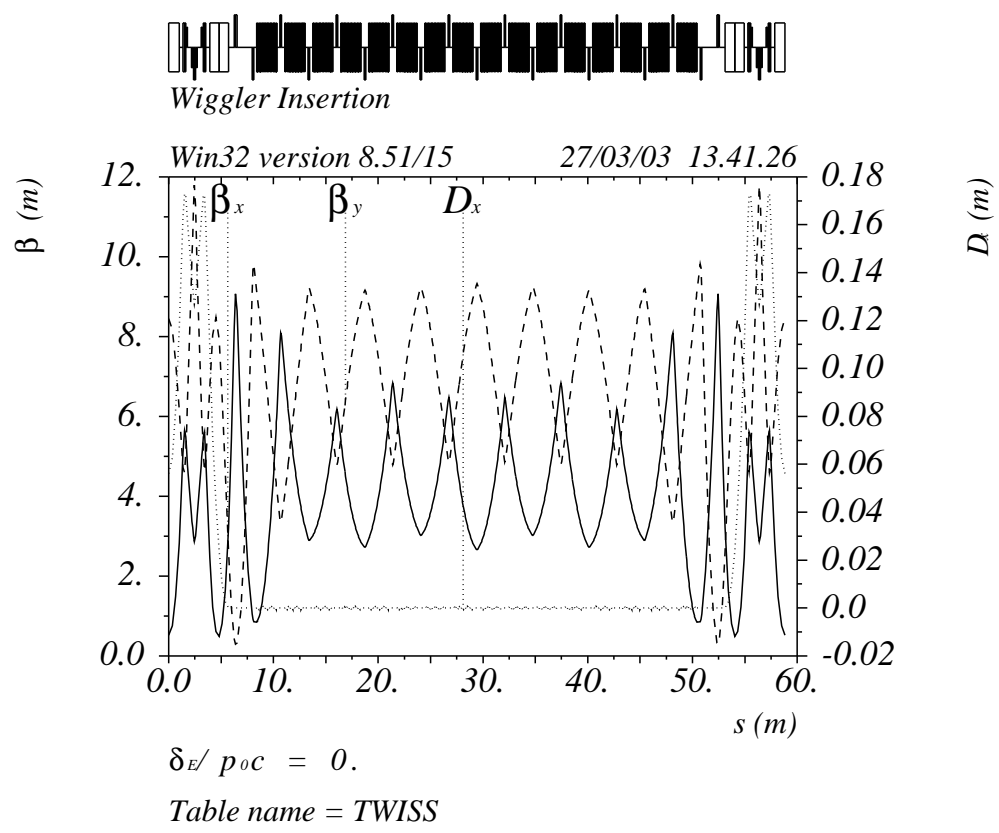

Figure 11: Lattice functions in the straight sections containing the wiggler insertions. Wigglers modelled using standard elements. Calculation carried out with MAD.

to the dynamics from a sextupole term in the field expansion does not average to zero. The integrated strength of this thin sextupole was chosen to correspond to the integral over halfwiggler period $\int_{0}^{\lambda_{w} / 2} C_{3}(z) d z=-7.78 \mathrm{~T} / \mathrm{m}$ of the generalized sextupole gradient $C_{3}(z)$ as determined in Sec. 4 with reversed sign at the two ends.

The second modification introduced in the lattice [1] consisted in changing the matching of the two main straight sections into the linear lattice to account for the different linear properties of our wiggler model. The new configuration was achieved by tuning the quadrupoles preceeding and trailing (Q1WM, Q2WM) or placed in between (Q3WM, Q4WM, QDW, QFW) the wiggler insertions so as to leave the lattice in the rest of the machine and tunes $\left(Q_{x}=21.150, Q_{y}=10.347\right)$ unaffected (see Table 3$)$. The matching was carried out with the MAD [10] fitting routines. In so doing we represented the lattice using the standard-element model of Sec. 8 not the realistic maps of Sec. 5 - this is reasonable as the linear parts of the maps in the two cases are very close to each other. The lattice functions in the main straight sections are shown in Fig. 11. As a check, in Fig. 12 we show the beta-functions that result from replacing the standard-elements in the wiggler body with the realistic maps and keeping the same matching scheme (plot made from MARYLiE output). The change in the lattice functions as well as in the machine tunes $\left(Q_{x}=21.152, Q_{y}=10.349\right)$ is minimal.

For the present study we switched off the rf cavities and did not include any errors in the magnets or wigglers - the combined effects of wiggler nonlinearities and systematic and random lattice errors will be the subject of further investigations in the future. Radiation effects were also neglected. Tracking was carried out element by element with MARYLiE3.0 using the symplectification of the corresponding $3^{\text {rd }}$-order maps. Symplectification in MARYLiE is done by the method of the generating functions, which requires solving some nonlinear algebraic equations to determine the positions and momenta of a particle orbit at the exit 


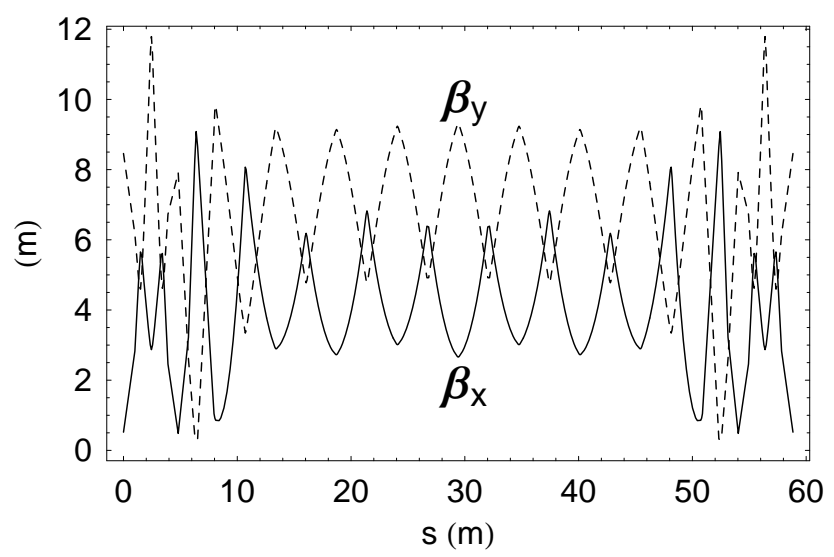

Figure 12: Beta-functions in the straight sections containing the wiggler insertions when the calculation is done using the GENMAP transfer maps to represent the wiggler periods (the rest of the ring lattice is left unchanged).
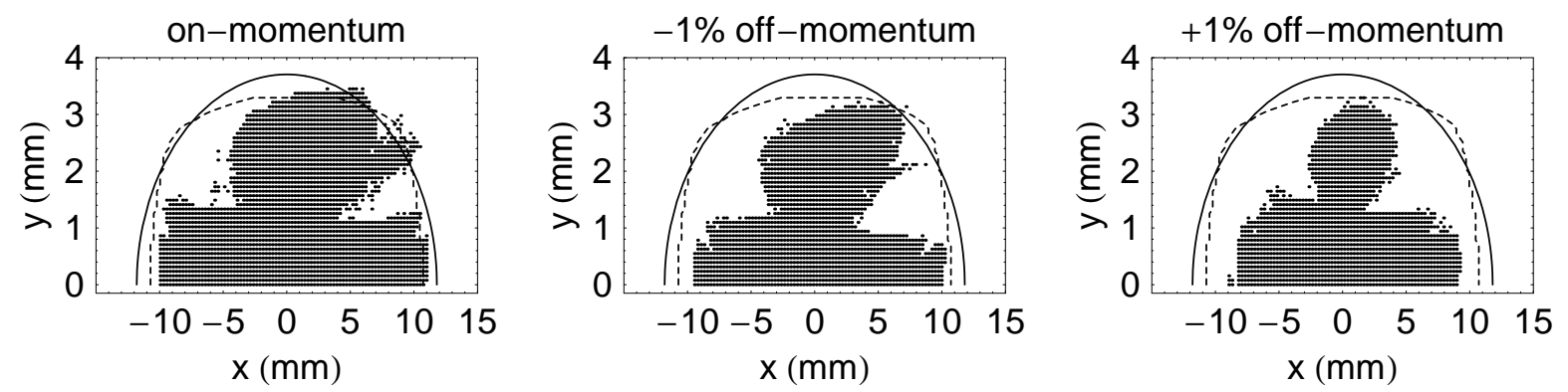

Figure 13: On and off-momentum DA for the NLC-MDR, using the GENMAP transfer maps for the wiggler periods through 500 machine turns. Orbits of particles touching the vacuum chamber wall in the wiggler insertions are marked as unstable. The solid line corresponds to a $15 \times \sigma$ transverse bunch size at injection, the dashed line to the transverse acceptance for a linear lattice corresponding to the $8 \mathrm{~mm}$ radius vacuum chamber aperture in the wigglers.

of each lattice element for given positions and momenta at the entrance. In some cases typically in the presence of significant nonlinearities - the Newton method used to solve the equations does not converge. We interpret this failure as an indication of the instability of the corresponding orbit [6]. Tracking through the body of the wiggler insertions was done by propagating the particle orbits through each individual period. When appropriate, the MARYLIE built-in modelling of fringe-field effects in the hard-edge limit was included in the representation of the magnet elements for the rest of the lattice. In this lattice, however, fringe fields are a negligible source of nonlinearities - the main contribution to the nonlinearities coming from the chromatic sextupoles and the wiggler insertions themselves.

The main result of this Section is represented by Fig. 13 showing the on and off-momentum DA in the transverse plane located in the middle of the first quadrupole magnet downstream the injection point (where $\beta_{x}=16.0 \mathrm{~m}, \beta_{y}=1.58 \mathrm{~m}, \alpha_{x}=-0.57$ and $\alpha_{y}=0.15$ ). Particles 
were launched with vanishing transverse momenta and tracked through 500 turns. We kept notice of the finite size of the circular cross section of the vacuum pipe in the wiggler insertion region (with radius $R=8 \mathrm{~mm}$ ) but not in the rest of the machine, where the radius of the pipe is larger $(R=20 \mathrm{~mm})$. Orbits of particles hitting the wall were marked as unstable. The corresponding transverse linear acceptance for the lattice is reported in Fig. 13 as a dashed line while the solid line indicates the $15 \sigma$ beam size at injection - a plausible DA target. Inspection of the figures shows that on-momentum a substantial portion of the available linear acceptance is indeed stable while some degradation of the DA is observed off-momentum.

The DA resulting when the presence of the vacuum chamber is not accounted for is shown on the left column of Fig. 14. In the same figure, the pictures to the right show the DA obtained by employing the standard-element model for the wiggler period. Comparison with the corresponding pictures on the left column shows a reasonable good agreement in the on-momentum case while off-momentum the standard-element model appears to gives a somewhat more pessimistic estimate of the DA. The DA estimates in the two cases however suggest that at least in preliminary calculations the use of the less refined model of wiggler dynamics may be justified.

Finally an assessment of the relative impact of the two main sources of nonlinearities chromatic sextupoles and wigglers - can be obtained by comparison of the two pictures of Fig. 15. The picture on the left shows the DA resulting from masking off the nonlinear part of the wiggler transfer maps so that the chromatic sextupoles dominate. In contrast, the DA in picture on the right was obtained by switching off the chromatic sextupoles leaving the wiggler nonlinearities to dominate.

\section{Conclusion}

Wiggler insertions contribute a significant amount of nonlinearities to the NLC-MDR lattice causing a reduction of the DA comparable to that produced by the chromatic sextupoles alone. The wiggler nonlinearities are prevalently of third order and result mostly from feeddown terms in the decapole component of the wiggler multipole field expansion. In turn, feed-down terms from the sextupole field component result in perturbation to the linear part of the dynamics. We determined the dynamical effects of the wigglers by integrating the corresponding transfer maps through the actual fields. For field representation we used a 3D multipole expansion derived from the field data obtained with the aid of a magnet design code.

We have compared the accurate maps so calculated to those relative to a simplified model of wiggler consisting of a sequence of standard magnet elements including thin octupoles to represent the dominating nonlinearity. By an appropriate choice of the magnet parameters we showed that the two mappings generate relatively close transverse kicks suggesting that the use of the less accurate map may be reasonable in a first assessment of wiggler dynamical effects.

Finally, we devised a possible compensation scheme for the wiggler nonlinearities consisting in placing an octupole magnet at each wiggler end - the strength of the two magnets 

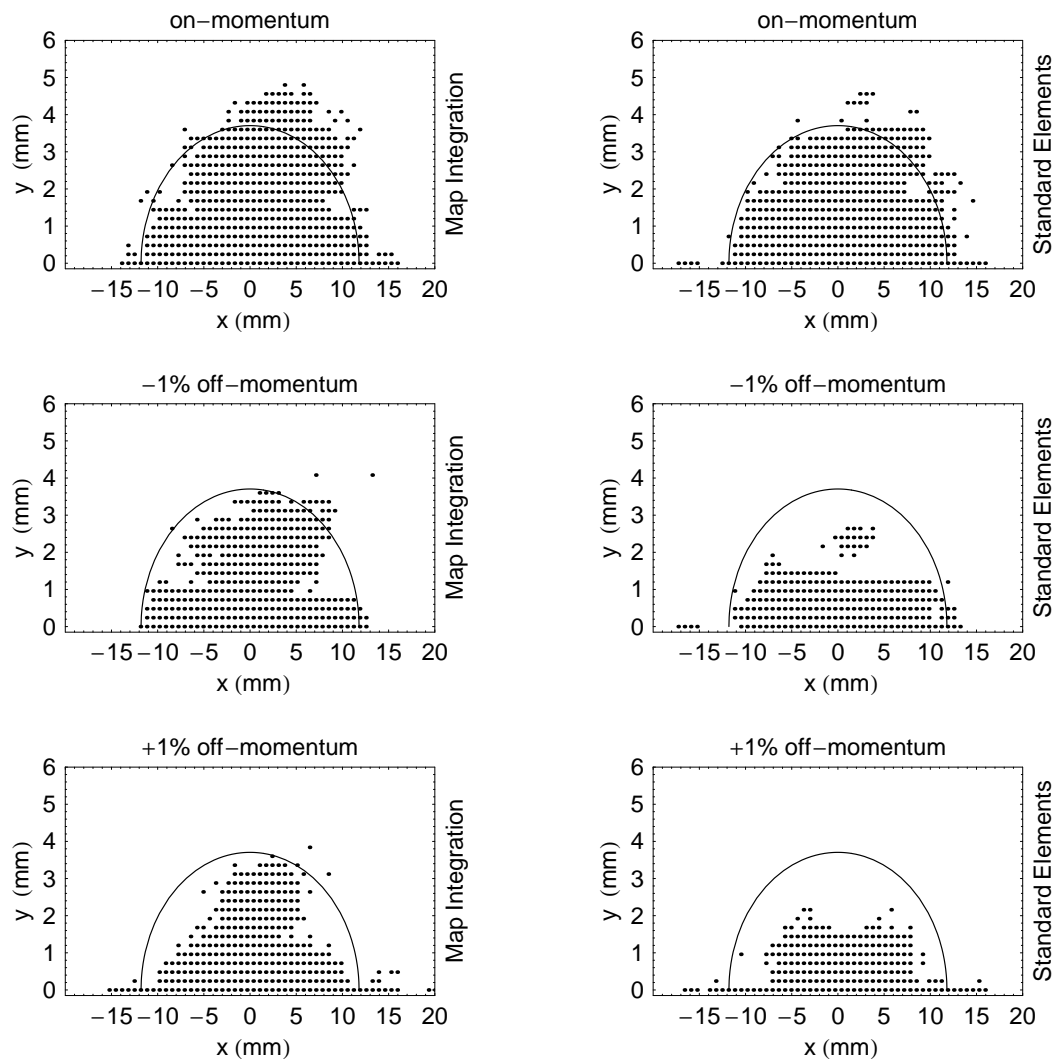

Figure 14: On and off-momentum Dynamic Aperture for the NLC-MDR, using wiggler transfer maps calculated using GENMAP (figures on the left) and standard element model (figures on the right). In this calculation the presence of vacuum-chamber wall is not accounted for.

being based on the fitting of the standard element model against the accurate map calculated from the realistic fields. Evaluation of the transverse kicks through a compensated insertion shows that the scheme is effective; however it remains to be determined whether this compensation may indeed have a significant impact on the DA of the NLC-MDR.

Work carried out in collaboration with A. Wolski and funded by DOE contract DE-AC0376SF00098.

\section{References}

[1] M. Woodley and A. Wolski, NLC Tech Note LCC-0113 (2003).

[2] M. Woodley, et al., A Lattice with Larger Momentum Compaction for the NLC Main Damping Rings PAC01, Proceedings, PAC03 Proceedings (2003). 

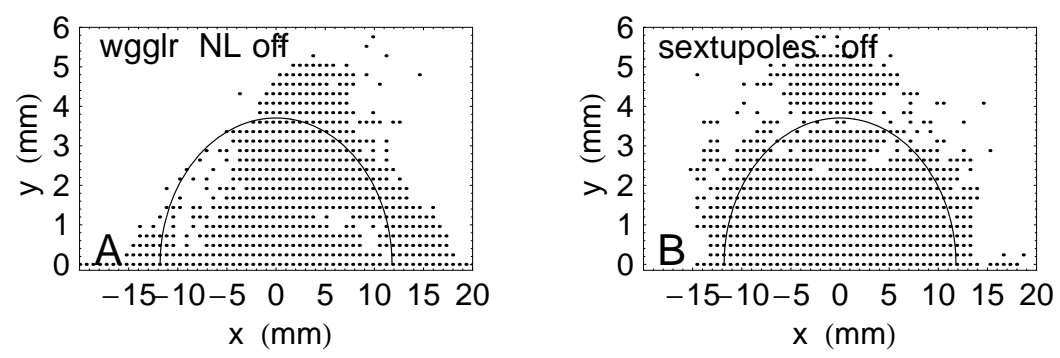

Figure 15: On-momentum Dynamic Aperture with wiggler nonlinearities turned off (A chromatic sextupoles dominate) and chromatic sextupoles turnded off (B - wiggler nonlinearities dominate).

[3] A. Wolski, Symplectic Integrators for Nonlinear Wiggler Fields, CBP Tech. Note-228, LCC-0062, Berkeley (2001).

[4] A. Wolski et al., PAC01 Proceedings, p. 3798 (2001).

[5] M. Venturini and A. Dragt, Nucl. Instr. and Meth. in Phys. Res. A, 415417 (1998).

[6] A. Dragt, et al., MARYLIE3.0, User's Manual, Dept. of Physics, University of Maryland (2002).

[7] J. Corlett et al., CBP Tech. Note 199, LBNL, Berkeley (1999), (also NLC Tech Note LCC-0031); S. Marks, private communication (2003).

[8] W. Decking, private communication.

[9] J. Safranek et al., Nonlinear Dynamics in SPEAR Wigglers, EPAC00, Proceedings (2000); Phys. Rev. ST-AB 5, 010701 (2002).

[10] H. Grote, The MAD Program CERN/SL/90-13 (AP).

\section{Appendix A: Lie Map For One NLC-MDR Wiggler Period - GENMAP Calculation.}

Below we report the wiggler one-period transfer map (in Lie form) for the NLC-MDR Damping ring as calculated by the GENMAP routine of MARYLIE3.0 starting from the magnetic field data. The linear part of the map is reported first as a $6 \times 6$ matrix. The ordering of the dynamical coordinates is $x, p_{x}, y, p_{y}, \tau, p_{\tau}$. Following is the list of the of the generators for the nonlinear part of the map. As for notation, $f(300000)$ refers to generator $x^{3}, f(210000)$ to generator $x^{2} p_{x}$, etc. The index on the left is used internally by MARYLIE for monomial book-keeping (Giorgilli index). 
matrix for map is :
$1.00046 \mathrm{E}+00$
$2.70086 \mathrm{E}-01 \quad 0.00000 \mathrm{E}+00$
$0.00000 \mathrm{E}+00$
$0.00000 E+00$
$9.71516 \mathrm{E}-08$
3. $37075 \mathrm{E}-03$
$1.00046 \mathrm{E}+00$
$0.00000 \mathrm{E}+00$
$0.00000 \mathrm{E}+00$
$0.00000 \mathrm{E}+00$
$1.66493 \mathrm{E}-10$
$0.00000 \mathrm{E}+00$
$0.00000 \mathrm{E}+00 \quad 9.97374 \mathrm{E}-01$
2.69792E-01
$0.00000 \mathrm{E}+00$
$0.00000 \mathrm{E}+00$
$0.00000 \mathrm{E}+00$
$0.00000 \mathrm{E}+00-1.94441 \mathrm{E}-02$
$9.97374 \mathrm{E}-01$
$0.00000 \mathrm{E}+00$
$0.00000 \mathrm{E}+00$
$1.60955 \mathrm{E}-10$
$9.71509 \mathrm{E}-08 \quad 0.00000 \mathrm{E}+00$
$0.00000 \mathrm{E}+00$
$1.00000 \mathrm{E}+00$
$2.95397 \mathrm{E}-05$
$0.00000 \mathrm{E}+00$
$0.00000 \mathrm{E}+00 \quad 0.00000 \mathrm{E}+00$
$0.00000 \mathrm{E}+00$
$0.00000 \mathrm{E}+00$
$1.00000 \mathrm{E}+00$

nonzero elements in generating polynomial are :

$f(28)=f\left(\begin{array}{lll}30 & 00 & 00\end{array}\right)=-2.05438713099290 \mathrm{E}-03$

$f\left(\begin{array}{lll}29\end{array}\right)=f\left(\begin{array}{lll}21 & 00 & 00\end{array}\right)=1.2191736272439$

$f(33)=f\left(\begin{array}{lll}20 & 00 & 01\end{array}\right)=1.68082035315380 \mathrm{E}-03$

$f(34)=f\left(\begin{array}{lll}12 & 00 & 00\end{array}\right)=-0.32913614569375$

$f(38)=f\left(\begin{array}{lll}11 & 00 & 01\end{array}\right)=1.19500647637689 \mathrm{E}-06$

$f\left(\begin{array}{ll}39\end{array}\right)=f\left(\begin{array}{lll}10 & 20 & 00\end{array}\right)=1.84722086028322 \mathrm{E}-02$

$f(40)=f\left(\begin{array}{lll}10 & 11 & 00\end{array}\right)=2.0751375008727$

$f(43)=f\left(\begin{array}{lll}10 & 02 & 00\end{array}\right)=-0.28065368212856$

$f(48)=f\left(\begin{array}{lll}10 & 00 & 02\end{array}\right)=2.46405198744314 \mathrm{E}-10$

$f(49)=f\left(\begin{array}{lll}03 & 00 & 00\end{array}\right)=3.04874328164547 \mathrm{E}-02$

$f(53)=f\left(\begin{array}{lll}02 & 00 & 01\end{array}\right)=-0.13506739929701$

$f\left(\begin{array}{ll}54\end{array}\right)=f\left(\begin{array}{lll}01 & 20 & 00\end{array}\right)=1.0358823246614$

$f(55)=f\left(\begin{array}{lll}01 & 11 & 00\end{array}\right)=-0.56044156015615$

$f\left(\begin{array}{lll}58\end{array}\right)=f\left(\begin{array}{lll}01 & 02 & 00\end{array}\right)=6.92114242109863 \mathrm{E}-02$

$f(63)=f\left(\begin{array}{lll}01 & 00 & 02\end{array}\right)=-2.41196416737200 E-07$

$f(67)=f\left(\begin{array}{llll}00 & 20 & 01\end{array}\right)=-9.72620828719118 \mathrm{E}-03$

$f(70)=f\left(\begin{array}{lll}00 & 11 & 01\end{array}\right)=-1.82475745603723 E-06$

$f\left(\begin{array}{ll}76\end{array}\right)=f\left(\begin{array}{lll}00 & 02 & 01\end{array}\right)=-0.13504241356563$

$f(83)=f\left(\begin{array}{lll}00 & 00 & 03\end{array}\right)=-1.47718808462429 \mathrm{E}-05$

$f(84)=f\left(\begin{array}{lll}40 & 00 & 00\end{array}\right)=-10.229319297423$

$f\left(\begin{array}{ll}85\end{array}\right)=f\left(\begin{array}{lll}31 & 00 & 00\end{array}\right)=5.5249661224250$

$f(89)=f\left(\begin{array}{lll}30 & 00 & 01\end{array}\right)=-2.05737316245644 \mathrm{E}-03$

$f(90)=f\left(\begin{array}{lll}22 & 00 & 00\end{array}\right)=-1.3000647075270$

$f(94)=f\left(\begin{array}{lll}21 & 00 & 01\end{array}\right)=1.2227198388800$

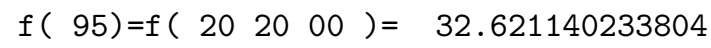

$f(96)=f\left(\begin{array}{lll}20 & 11 & 00\end{array}\right)=-8.8079626057123$

$f(99)=f\left(\begin{array}{lll}20 & 02 & 00\end{array}\right)=0.96147043624757$

$f(104)=f\left(\begin{array}{lll}20 & 00 & 02\end{array}\right)=1.67436358151190 \mathrm{E}-03$

$f(105)=f\left(\begin{array}{lll}13 & 00 & 00\end{array}\right)=0.14985505766427$

$f(109)=f\left(\begin{array}{lll}12 & 00 & 01\end{array}\right)=-0.33017730792798$

$f(110)=f\left(\begin{array}{lll}11 & 20 & 00\end{array}\right)=-8.7944794469442$

$f(111)=f\left(\begin{array}{lll}11 & 11 & 00\end{array}\right)=3.9199892653524$

$f(114)=f\left(\begin{array}{lll}11 & 02 & 00\end{array}\right)=-0.46788275619052$

$f(119)=f\left(\begin{array}{lll}11 & 00 & 02\end{array}\right)=3.16513794572837 \mathrm{E}-06$

$f(123)=f\left(\begin{array}{lll}10 & 20 & 01\end{array}\right)=1.84050731398390 \mathrm{E}-02$

$f(126)=f\left(\begin{array}{lll}10 & 11 & 01\end{array}\right)=2.0678251020578$

$f(132)=f\left(\begin{array}{lll}10 & 02 & 01\end{array}\right)=-0.27970654678607$

$f(139)=f\left(\begin{array}{lll}10 & 00 & 03\end{array}\right)=5.70944853141676 E-10$

$f(140)=f\left(\begin{array}{lll}04 & 00 & 00\end{array}\right)=-4.21008925399704 \mathrm{E}-02$

$f(144)=f\left(\begin{array}{lll}03 & 00 & 01\end{array}\right)=4.71172146159332 \mathrm{E}-02$ 


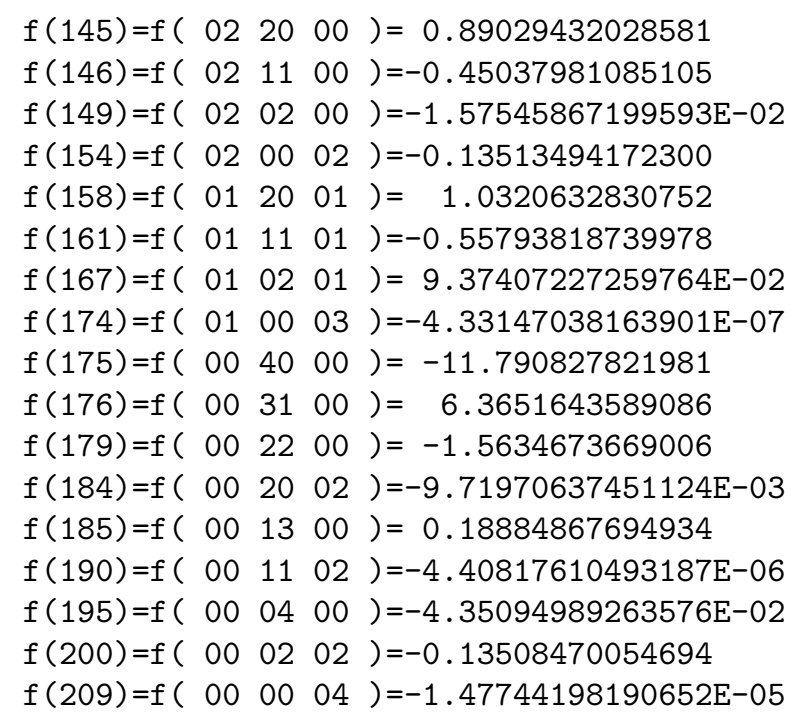

The quality of the numerical integration can be assessed by checking on the periodicity of the reference orbit, through a wiggler period. The data below show that differences between initial and final positions and angles is of the order of $10^{-9}$ ( $\mathrm{m}$ and rad, respectively).

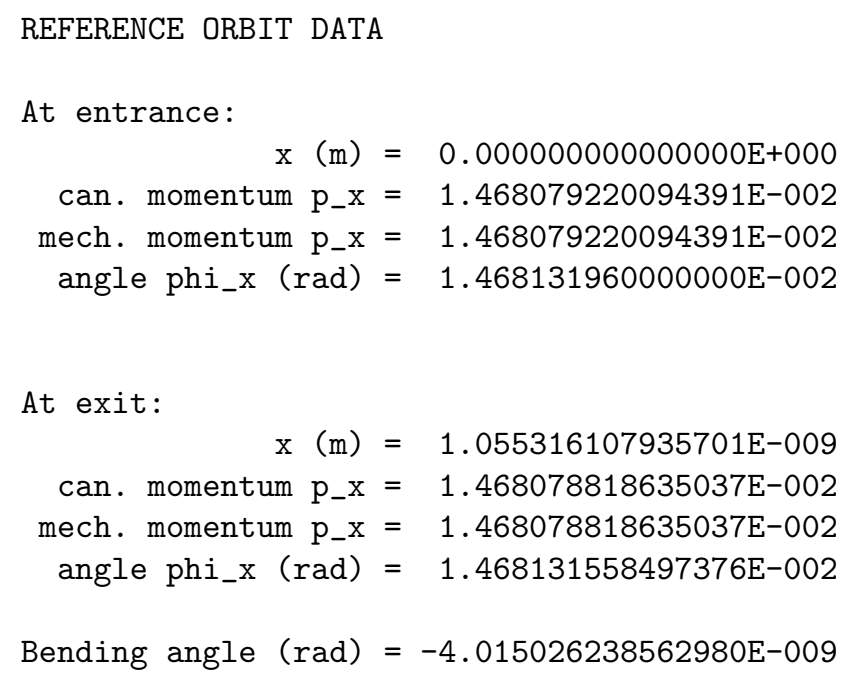

\section{Appendix B: Lie Map For One NLC-MDR Wiggler Period - Standard Elements}

We report excerpts from the MARYLIE input file used to perform the calculation of the transfer map for one NLC-MDSR wiggler-period using standard elements. In MARYLIE combined function sector bends (with optional additional higher order multipoles) are set up using the type code 'cfbd'. The map for rectangular bends can be obtained by combining 'cfbd' with the 'Body of a General Bending Magnet' (gbdy), called with vanishing bending angle but finite entry or exit angle. This is done in the lines 'bwpu', 'bwpd', 'bwmu', 'bwmd', 
defining the first halves and second halves of the wiggler poles with positive ('bwpu', 'bwpd') and negative ('bwmu', 'bwmd') polarity. The same lines also include accounts for fringe effects (type code 'frng') and a required rotation of the transverse planes (type cose 'prot'). In the code MAD [10] the same action is achieved by use of the physical element 'SBEND' with appropriate setting of the entrance and exit angles for the magnet faces.

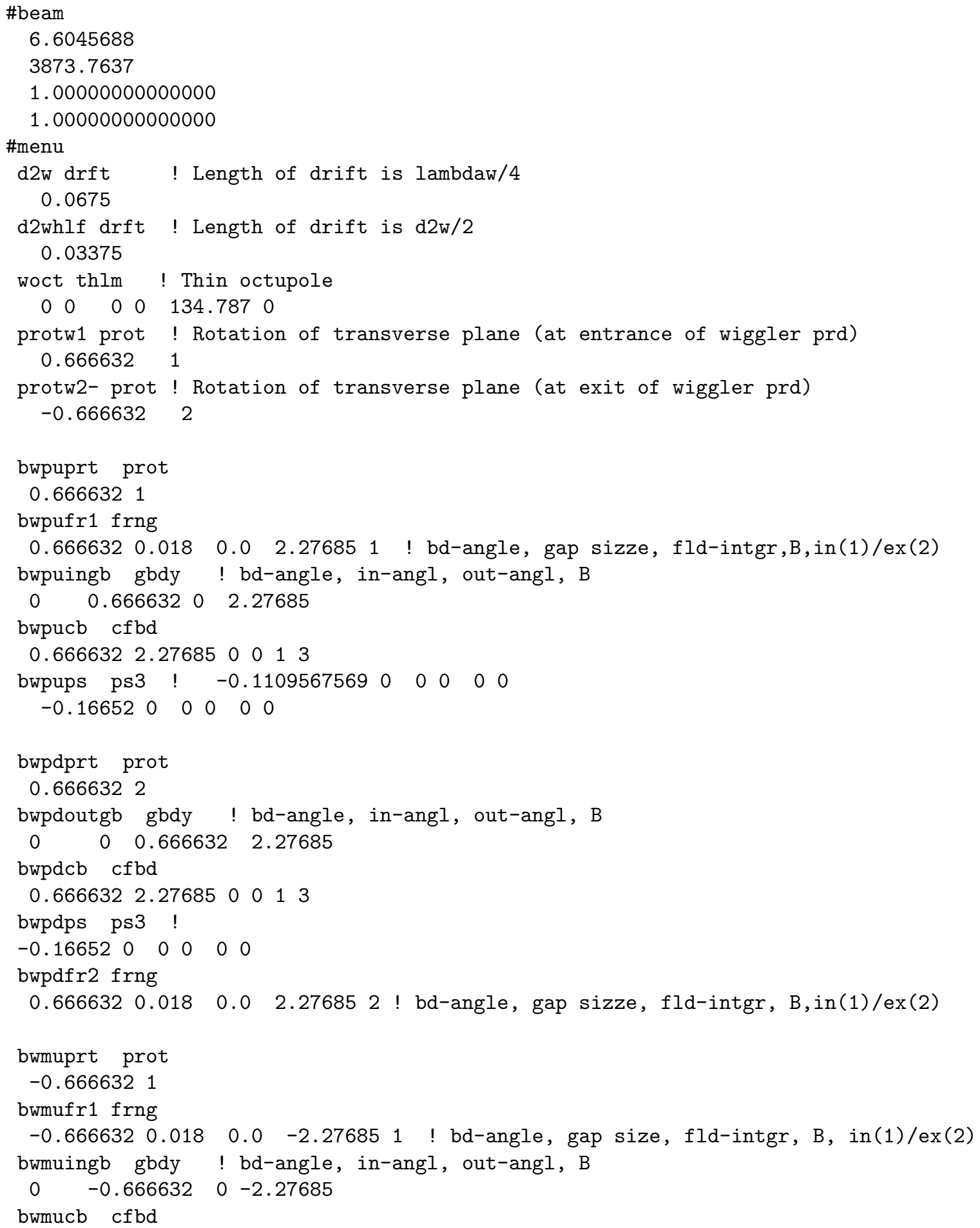




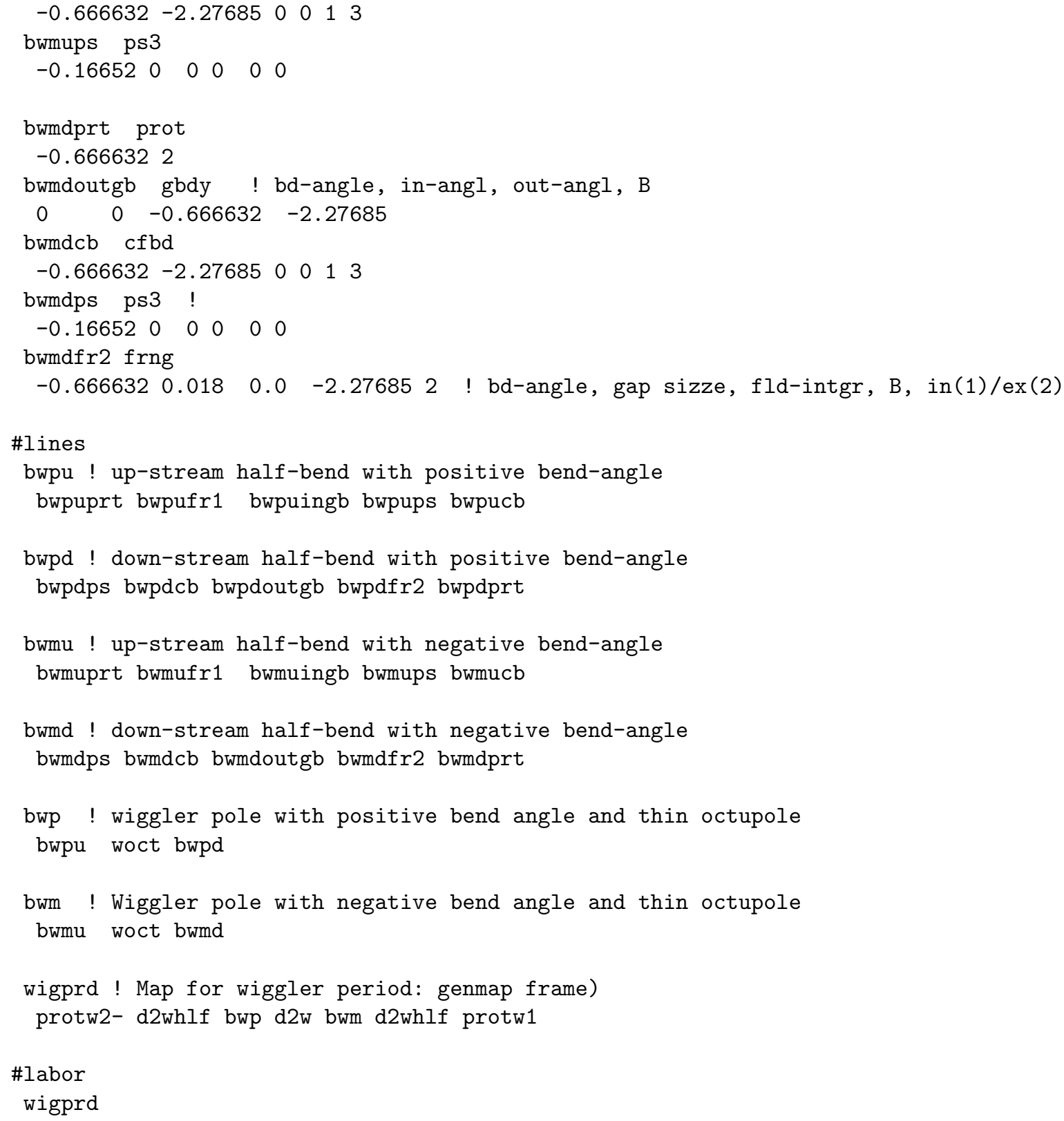

The commands 'protw2-' and 'protw1' in line 'wigprd' cause the rotation of the transverse planes at both wiggler-period ends required for a proper comparison with the map calculated using GENMAP and reported in Appendix A (see end of Section 8 for further comments on this point). The transfer map written out in response to the 'wigprd' command under \#labor corresponding to a NLC-MDR one wiggler period reads:

matrix for map is :
$1.00046 \mathrm{E}+00 \quad 2.70070 \mathrm{E}-01$
$0.00000 \mathrm{E}+00$
$0.00000 \mathrm{E}+00$
$0.00000 \mathrm{E}+00 \quad 1.20325 \mathrm{E}-07$
$3.40404 \mathrm{E}-03$
$1.00046 \mathrm{E}+00$
$0.00000 \mathrm{E}+00$
$0.00000 \mathrm{E}+00$
$0.00000 \mathrm{E}+00$
$2.04749 \mathrm{E}-10$ 

$0.00000 \mathrm{E}+00$
$0.00000 \mathrm{E}+00 \quad 9.97376 \mathrm{E}-01$
$2.69754 \mathrm{E}-01$
$0.00000 \mathrm{E}+00$
$0.00000 \mathrm{E}+00$
$0.00000 \mathrm{E}+00 \quad 0.00000 \mathrm{E}+00-1.94326 \mathrm{E}-02$
$9.97376 \mathrm{E}-01$
$0.00000 \mathrm{E}+00$
$0.00000 \mathrm{E}+00$
$2.04749 \mathrm{E}-10$
$1.20325 \mathrm{E}-07 \quad 0.00000 \mathrm{E}+00$
$0.00000 \mathrm{E}+00$
$1.00000 \mathrm{E}+00$
$2.43859 \mathrm{E}-05$
$0.00000 \mathrm{E}+00$
$0.00000 \mathrm{E}+00$
$0.00000 \mathrm{E}+00$
$0.00000 \mathrm{E}+00$
$0.00000 \mathrm{E}+00$
$1.00000 \mathrm{E}+00$

nonzero elements in generating polynomial are :

$f(28)=f\left(\begin{array}{lll}30 & 00 & 00\end{array}\right)=5.05678861701021 E-08$

$f(29)=f\left(\begin{array}{lll}21 & 00 & 00\end{array}\right)=-2.97310321376835 \mathrm{E}-05$

$f(33)=f\left(\begin{array}{lll}20 & 00 & 01\end{array}\right)=-4.77399800909765 \mathrm{E}-07$

$f(34)=f\left(\begin{array}{lll}12 & 00 & 00\end{array}\right)=8.02635311854781 \mathrm{E}-06$

$f(38)=f\left(\begin{array}{lll}11 & 00 & 01\end{array}\right)=4.59782341313606 \mathrm{E}-04$

$f(39)=f\left(\begin{array}{lll}10 & 20 & 00\end{array}\right)=-1.64635584781963 \mathrm{E}-06$

$f(40)=f\left(\begin{array}{lll}10 & 11 & 00\end{array}\right)=-1.38027108546669 \mathrm{E}-04$

$f(43)=f\left(\begin{array}{lll}10 & 02 & 00\end{array}\right)=2.40238060335417 \mathrm{E}-05$

$f(48)=f\left(\begin{array}{lll}10 & 00 & 02\end{array}\right)=4.09624350058919 \mathrm{E}-10$

$f(49)=f\left(\begin{array}{lll}03 & 00 & 00\end{array}\right)=-8.38791410965890 \mathrm{E}-07$

$f(53)=f\left(\begin{array}{lll}02 & 00 & 01\end{array}\right)=-0.13509397189996$

$f(54)=f\left(\begin{array}{lll}01 & 20 & 00\end{array}\right)=1.76433099074306 \mathrm{E}-04$

$f(55)=f\left(\begin{array}{lll}01 & 11 & 00\end{array}\right)=-9.00750112029660 \mathrm{E}-05$

$f(58)=f\left(\begin{array}{lll}01 & 02 & 00\end{array}\right)=-3.13666154900816 \mathrm{E}-03$

$f(63)=f\left(\begin{array}{llll}01 & 00 & 02\end{array}\right)=-2.40719038192144 \mathrm{E}-07$

$f(67)=f\left(\begin{array}{lll}00 & 20 & 01\end{array}\right)=-8.02747907582517 \mathrm{E}-03$

$f(70)=f\left(\begin{array}{lll}00 & 11 & 01\end{array}\right)=-4.58337972064577 \mathrm{E}-04$

$f\left(\begin{array}{lll}76\end{array}\right)=f\left(\begin{array}{lll}00 & 02 & 01\end{array}\right)=-0.13495786239198$

$f(83)=f\left(\begin{array}{lll}00 & 00 & 03\end{array}\right)=-1.21939513062731 \mathrm{E}-05$

$f(84)=f\left(\begin{array}{lll}40 & 00 & 00\end{array}\right)=-10.209368709268$

$f(85)=f\left(\begin{array}{lll}31 & 00 & 00\end{array}\right)=5.5141621205550$

$f(89)=f\left(\begin{array}{lll}30 & 00 & 01\end{array}\right)=-5.78127029741315 \mathrm{E}-06$

$f(90)=f\left(\begin{array}{lll}22 & 00 & 00\end{array}\right)=-1.3958594964358$

$f(94)=f\left(\begin{array}{lll}21 & 00 & 01\end{array}\right)=4.84224087575559 \mathrm{E}-03$

$f(95)=f\left(\begin{array}{lll}20 & 20 & 00\end{array}\right)=61.141659215930$

$f\left(\begin{array}{l}96) \\ f\end{array}\right)\left(\begin{array}{lll}20 & 11 & 00\end{array}\right)=-16.511332528575$

$f(99)=f\left(\begin{array}{lll}20 & 02 & 00\end{array}\right)=1.3940721611217$

$f(104)=f\left(\begin{array}{lll}20 & 00 & 02\end{array}\right)=-2.17247492280703 E-05$

$f(105)=f\left(\begin{array}{lll}13 & 00 & 00\end{array}\right)=0.17610414850026$

$f(109)=f\left(\begin{array}{lll}12 & 00 & 01\end{array}\right)=-1.30712367374559 E-03$

$f(110)=f\left(\begin{array}{lll}11 & 20 & 00\end{array}\right)=-16.498746307302$

$f(111)=f\left(\begin{array}{lll}11 & 11 & 00\end{array}\right)=5.5711828001835$

$f(114)=f\left(\begin{array}{lll}11 & 02 & 00\end{array}\right)=-0.52668050761575$

$f(119)=f\left(\begin{array}{lll}11 & 00 & 02\end{array}\right)=4.65782757334236 E-04$

$f(123)=f\left(\begin{array}{lll}10 & 20 & 01\end{array}\right)=-9.71124593912131 \mathrm{E}-05$

$f(126)=f\left(\begin{array}{lll}10 & 11 & 01\end{array}\right)=-9.96662980618190 \mathrm{E}-03$

$f(132)=f\left(\begin{array}{lll}10 & 02 & 01\end{array}\right)=1.36386651273774 \mathrm{E}-03$

$f(139)=f\left(\begin{array}{lll}10 & 00 & 03\end{array}\right)=6.16244292868278 E-10$

$f(140)=f\left(\begin{array}{lll}04 & 00 & 00\end{array}\right)=-4.24851093382762 E-02$

$f(144)=f\left(\begin{array}{lll}03 & 00 & 01\end{array}\right)=9.44878235051379 \mathrm{E}-05$

$f(145)=f\left(\begin{array}{lll}02 & 20 & 00\end{array}\right)=1.3714816322383$

$f(146)=f\left(\begin{array}{lll}02 & 11 & 00\end{array}\right)=-0.52229761226595$

$f(149)=f\left(\begin{array}{lll}02 & 02 & 00\end{array}\right)=-1.58645779384552 \mathrm{E}-02$

$f(154)=f\left(\begin{array}{lll}02 & 00 & 02\end{array}\right)=-0.13517196677457$

$f(158)=f\left(\begin{array}{lll}01 & 20 & 01\end{array}\right)=-4.29896700468791 E-03$ 


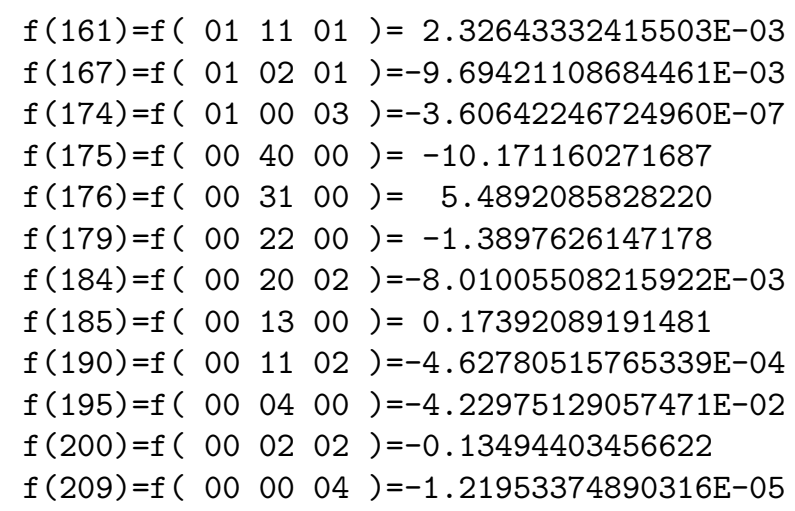

\section{Appendix C: Multipole Representation for an Ideal Wiggler with Infinite Width}

Because ideal wigglers with infinite width are often used in elementary discussions of beam dynamics it may be of interest to report the 3D multipole representation for the corresponding field configuration. Having denoted with $x$ the direction along which the system is translational invariant we have $B_{x}=0$, while the other two components of the magnetic field - having chosen a sinusoidal dependence along the $z$ - axis - read

$$
\begin{aligned}
& B_{y}=B_{0} \cos \left(\frac{2 \pi}{\lambda_{w}} z\right) \cosh \left(\frac{2 \pi}{\lambda_{w}} y\right) \\
& B_{z}=-B_{0} \sin \left(\frac{2 \pi}{\lambda_{w}} z\right) \sinh \left(\frac{2 \pi}{\lambda_{w}} y\right) .
\end{aligned}
$$

The generalized gradients for this field can be easily determined upon writing the radial component of the magnetic field

$$
B_{\rho}=B_{y} \sin \phi=B_{0} \cos \left(\frac{2 \pi}{\lambda_{w}}\right) \cosh \left(\frac{2 \pi}{\lambda_{w}} \rho \sin \phi\right) \sin \phi
$$

Expansion of (44) in power series of $\rho$ (having set $\xi=2 \pi / \lambda_{w}$ )

$$
\begin{aligned}
B_{\rho} & =B_{0} \cos (\xi z)\left[\left(1+\frac{3}{8} \rho^{2} \xi^{2}+\frac{5}{192} \rho^{4} \xi^{4}+\ldots\right) \sin \phi\right. \\
& \left.-\left(\frac{1}{8} \rho^{2} \xi^{2}+\frac{5}{384} \rho^{4} \xi^{4}+\ldots\right) \sin (3 \phi)+\frac{1}{384} \rho^{4} \xi^{4} \sin 5(\phi)+\ldots\right]
\end{aligned}
$$

and comparison with the general expression for the 3D multipole expansion of $B_{\rho}$ (first line in Eq. 38) allow us to identify the generalized gradients as

$$
C_{m}(z)=B_{0} \frac{(-1)^{(m-1) / 2}}{m ! 2^{m-1}}\left(\frac{2 \pi}{\lambda_{w}}\right)^{m-1} \cos \left(\frac{2 \pi}{\lambda_{w}} z\right)
$$


for odd $m$. For even $m, C_{m}(z)=0$. Notice that while an infinite number of modes appear in the spectrum of the 3D multipole expansion their magnitude decreases very rapidly with the order $m$ of the multipole.

Using the above equation it is easy to verify that for an infinitely wide wiggler the combinations $-\frac{1}{4} C_{1}^{[2]}(z) d z-6 C_{3}(z)$ and $\frac{C_{1}^{[4]}(z)}{48}-\frac{3 C_{3}^{[2]}(z)}{4}+20 C_{5}(z)$ entering in the expressions (29) and (30) for the horizontal kicks vanish (by inspection of Expression (38) one also recognizes that this is equivalent to the property that the vertical component $B_{y}$ of the magnetic field have no roll-off in $x$ ). That is, in the variables expressing deviation from the reference orbit the particle dynamic in the horizontal plane is that of a drift - as expected. Notice that Eqs. (29) and (30) which in general are only approximately valid, are exact for a infinitely wide wiggler. 
This document was prepared as an account of work sponsored by the United States Government. While this document is believed to contain correct information, neither the United States Government nor any agency thereof, nor The Regents of the University of California, nor any of their employees, makes any warranty, express or implied, or assumes any legal responsibility for the accuracy, completeness, or usefulness of any information, apparatus, product, or process disclosed, or represents that its use would not infringe privately owned rights. Reference herein to any specific commercial product, process, or service by its trade name, trademark, manufacturer, or otherwise, does not necessarily constitute or imply its endorsement, recommendation, or favoring by the United States Government or any agency thereof, or The Regents of the University of California. The views and opinions of authors expressed herein do not necessarily state or reflect those of the United States Government or any agency thereof, or The Regents of the University of California.

Ernest Orlando Lawrence Berkeley National Laboratory is an equal opportunity employer. 